\title{
IDENTIFICATION OF NITRIDING MECHANISMS IN High Purity Reaction Bonded Silicon Nitride
}

by:

John S. Haggerty

Massáchusetts Institute of Technology

Room 12-011

77 Massachusetts Avenue

Cambridge MA 02139-4307

Research Performed for:

Dr. Alan L. Dragoo

U.S. Department of Energy

Division of Materials Science

19901 Germantown Road

Germantown, MD 20874 


\title{
Table of Contents
}

Page

SUMMARY OF PRINCIPAL FINDINGS 2

I. INTRODUCTION AND BACKGROUND 5

II. NITRIDING KINETICS OF HIGH PURITY SILICON POWDERS 8

A. Materials and Experimental Procedures 8

B. Macroscopic Nitriding Kinetics 10

C. Nitrogen Adsorption on Silicon Powder Surfaces 15

D. $\mathrm{Si}_{3} \mathrm{~N}_{4}$ Nucleation and Growth 16

E. Rapid Growth and Complete Conversion 24

F. The Effects of Low-Level Oxygen Contamination 26

G. The Effects of Binder and Solvent Exposures 28

H. Microstructural Characterizations 31

III. CONCLUSIONS 34

$\begin{array}{ll}\text { REFERENCES } & 35\end{array}$

Attachment I. List of Publications Resulting in part from DOE Support

\section{DISCLAIMER}

\begin{abstract}
This report was prepared as an account of work sponsored by an agency of the United States Government. Neither the United States Government nor any agency thereof, nor any of their employees, makes any warranty, express or implied, or assumes any legal liability or responsibility for the accuracy, completeness, or usefulness of any information, apparatus, product, or process disclosed, or represents that its use would not infringe privately owned rights. Reference herein to any specific commercial product, process, or service by trade name, trademark, manufacturer, or otherwise does not necessarily constitute or imply its endorsement, recommendation, or favoring by the United States Government or any agency thereof. The views and opinions of authors expressed herein do not necessarily state or reflect those of the United States Government or any agency thereof.
\end{abstract}




\title{
Identification of Nitriding Mechanisms in High Purity Reaction Bonded Silicon Nitride
}

\author{
Grant No. DE-FG02-87ER45313 \\ Principal Investigator: Dr. John S. Haggerty \\ Organization: Massachusetts Institute of Technology \\ Address: Room 12-011 \\ 77 Massachusetts Avenue \\ Cambridge MA 02139-4307
}

\section{Summary of PRINCIPAL FINDINGS}

The principal objective of this nitriding research was the definition of processing conditions that would enable the full potential of reaction bonded silicon nitride to be realized. Traditor ally, enhanced processibility has been sought at the expense of properties. Nitriding mechanism research conducted at MIT focused on defining the relationships between nucleation and growth mechanisms and the evolving microstructures. Experiments simplified the nitriding process by identifying processing conditions where thermal and concentration gradients were minimized, and by using simple, high-purity reactants ( $\mathrm{Si}$ and $\mathrm{N}_{2}$ ). MIT's unique capability to produce very high-purity, $\mathrm{SiH}_{4}$-derived $\mathrm{Si}$ powders that are both unagglomerated and spherical was an essential element of this program's success.

The results of this research with the high purity Si powders can be summarized by the following major findings:

1. Without employing property degrading nitriding accelerators, complete conversion to $\mathrm{Si}_{3} \mathrm{~N}_{4}$ can be obtained at relatively low temperatures and in unusually short times (e.g., $1150^{\circ} \mathrm{C}$ for $1 \mathrm{~h}$ or $1250^{\circ} \mathrm{C}$ for $\left.10 \mathrm{~m}\right)$. The use of pre-nucleation heat treatments can reduce the required temperature to $1050^{\circ} \mathrm{C}$.

2. The macroscopic nitriding kinetics can be described as three sequential periods: (1) an induction period where very little nitridation occurs, (2) a "fast" reaction period where nitridation is extremely rapid and where complete conversion to $\mathrm{Si}_{3} \mathrm{~N}_{4}$ can be obtained at relatively low temperatures, and (3) a "slow" reaction period where nitridation is not as rapid, and where complete conversion can only be obtained at higher temperatures (above $1350^{\circ} \mathrm{C}$ ) if the slow period commences prematurely.

3. During the induction period nitrogen reacts slowly to form a thin amorphous layer on the Si particles, while small amounts of surface oxygen (submonolayer) are also apparently removed.

4. The nucleation of crystalline $\mathrm{Si}_{3} \mathrm{~N}_{4}$ coincides with the onset of the "fast" reaction period. Transmission electron microscopy indicates that 
nucleation is accompanied by a rearrangement of the initial spherical particle surfaces to form essentially flat $\mathrm{Si} / \mathrm{Si}_{3} \mathrm{~N}_{4}$ interfaces. Several different crystallographic relationships between the $\mathrm{Si}$ and the $\mathrm{Si}_{3} \mathrm{~N}_{4}$ have been identified.

5. Growth during the fast reaction period occurs by vapor phase transport of Si from Si-vapor interfaces on the particle surfaces remaining between growing $\mathrm{Si}_{3} \mathrm{~N}_{4}$ grains.

6. Premature cessation of the "fast" reaction prior to complete nitridation apparently occurs when the number of nuclei that form per particle is too low. The details of the transition from the "fast" reaction period to the "slow" reaction period have not been fully described; however, it appears that incomplete nitridation occurs when preferred growth planes impinge on surrounding material and limit additional rapid nitridation.

7. Small changes in the Si surface chemistry have dramatic effects on the nitriding kinetics. Specifically, small submonolayer amounts of oxygen inhibit nucleation and limit nitridation. Small amounts of excess carbon can apparently mitigate the effects of oxygen, while various hydrocarbons have a wide range of subtler effects, some of which are detrimental and some of which are not.

8. The initial $\mathrm{Si}-\mathrm{H}$ surface of the powders transforms to an $\mathrm{Si}-\mathrm{N}$ surface between 700 and $1000^{\circ} \mathrm{C}$ (i.e., before the sample reaches the nitriding temperature). In general, the surface reactions at low and moderate temperatures have a strong influence on the nucleation of $\mathrm{Si}_{3} \mathrm{~N}_{4}$ at higher temperatures, that ultimately controls the RBSN microstructure.

Although the three reaction periods described in item 2 are superficially similar to those reported by others (e.g. Moulson's excellent review ${ }^{1}$ ), the nitriding mechanisms observed with the high purity powders are significantly different from previously reported concepts in the following ways:

1. The induction period observed elsewhere has been attributed to the removal of the native oxide layer on the Si. The silane derived powders have no native oxide layer, which is one of the primary reasons that nitridation can be obtained at lower temperatures. Without this native oxide, the induction period observed with these powders is significantly different, involving a slow nitridation reaction, the desorption of submonolayer amounts of oxygen, and some faceting prior to nucleation. These simultaneous processes are much more complex than Moulson's ${ }^{1}$ relatively simple active oxidation concept.

2. The observations that nucleation occurs only after some rearrangement of the initial surface, and that only certain heteroepitaxial relationships exist, indicate that nucleation is strongly affected simultaneously by the crystallography as well as the macroscopic surface morphology of the Si powders. Previous work ${ }^{2}$ on crystallographic relationships between 
the two phases did not present TEM results, so it is difficult to assess. Also, previous research addressed surface features that are much smaller than the Si particles, so it would not have considered surface curvature effects. The geometric evolution of the microstructure is very different for the high purity RBSN because a much smaller number of $\mathrm{Si}_{3} \mathrm{~N}_{4}$ nuclei per Si particle are allowed to form.

3. The rapid nitridation described by Moulson ${ }^{1}$ is much slower than the "fast" reaction observed with the high purity Si powders, such that the "slow" reaction rates are actually closer to Moulson's rapid rates. This is largely due to differences in the Si powders. Also, Moulson attributes the end of rapid nitridation to coverage of the Si/vapor surfaces, which is completely different from the mechanism that has been suggested for the silane derived powders.

4. In contrast to nitriding mechanisms and resulting microstructures reported for conventional Si powders, both $\alpha$ - and $\beta-\mathrm{Si}_{3} \mathrm{~N}_{4}$ phases nucleate simultaneously in the high purity materials and grow at identical rates by the same vapor phase mechanism. This difference results in equal-diameter equiaxed $\alpha$ - and $\beta-\mathrm{Si}_{3} \mathrm{~N}_{4}$ grains in the high purity RBSN instead of the relatively small $\alpha-\mathrm{Si}_{3} \mathrm{~N}_{4}$ needles that form via the vapor phase and larger equiaxed $\beta-\mathrm{Si}_{3} \mathrm{~N}_{4}$ grains that form via a liquid in conventional RBSN.

This fundamental nitriding kinetic research has identified reliable means to convert green $\mathrm{Si}$ parts to very high quality $\mathrm{Si}_{3} \mathrm{~N}_{4}$ using temperatures and times that enable composites to be made without damaging reinforcements during the reaction period and without overheating large parts. Realizing strengths up to $870 \mathrm{MPa}$, specific strengths (up to $0.36 \mathrm{MPa} /\left(\mathrm{kg} / \mathrm{m}^{3}\right)$ ), strain to failure values up to $0.3 \%$, oxidation resistance up to 20 times better than fully dense hot pressed (HPSN) or sintered (SSN) $\mathrm{Si}_{3} \mathrm{~N}_{4}$, full strength retention after oxidation, and dimensional stabilities of $\sim 0.1-2.0 \%$ linear change from die to nitrided part are all improvements that were made possible only by understanding the nitriding mechanisms. These findings have also provided the basis for achieving similar results for RBSN parts made with ceramic forming and handling techniques that appear rather conventional, but avoid critical exposures. 


\section{INTRODUCTION AND BACKGROUND}

Reaction bonded $\mathrm{Si}_{3} \mathrm{~N}_{4}$ is made by heating a porous $\mathrm{Si}$ preform, typically a green Si powder compact, in a nitriding gas, which is usually $\mathrm{N}_{2}, a \mathrm{~N}_{2} / \mathrm{H}_{2}$ mixture, or $\mathrm{NH}_{3}$, until it is converted to $\mathrm{Si}_{3} \mathrm{~N}_{4}$. Commercial grades of RBSN typically retain a few percent of unreacted $\mathrm{Si}$, which precludes them from being candidates for applications for which they are otherwise well suited. The $22 \%$ volumetric increase caused by the nitridation reaction occurs within the void space of the compact, ${ }^{1}$ so a Si body that is $65 \%$ dense will form RBSN that is $79 \%$ dense. This simple process gives a rapid means of making high-purity, net-shape parts because no significant dimensional change is caused by the reaction and no sintering aids or other impurities need to be introduced to the material. Relatively high levels of residual porosity are characteristic of RBSN because an interconnected pore structure is required to allow transport of $\mathrm{N}_{2}$ throughout the part, and because maximum green packing densities of the $\mathrm{Si}$ particles will usually leave voids. Until very recently, nitriding schedules needed to insure complete reaction have involved temperatures that exceed the melting point of $\mathrm{Si}$ (up to $1550^{\circ} \mathrm{C}$ ) and times up to 150 hours. ${ }^{3-10}$

Traditionally, RBSN processing research has concentrated on identifying additives that enhance the reaction kinetics (typically iron, nickel and cobalt oxides), avoiding the adverse effects of excessive temperatures that result from the highly exothermic reactions, and introducing sintering aids (typically combinations of $\mathrm{MgO}, \mathrm{Al}_{2} \mathrm{O}_{3}$ and $\mathrm{Y}_{2} \mathrm{O}_{3}$ ) that allow the reacted parts to be densified by conventional sintering or pressure assisted processes. Although seemingly well founded from a conventional ceramics viewpoint, the use of additives to either enhance reaction kinetics or induce densification results in a material that loses much of its intrinsic potential. The additives concentrate in grain boundaries where they degrade both high temperature properties and resistance to chemical attack, while the post-nitriding densification gives up the potential net-shape advantage since the as-nitrided parts have about the same density levels as is achieved in an optimized powder compact.

MIT's RBSN research has been carried out using Si powders derived from $\mathrm{CO}_{2}$ laser heated $\mathrm{SiH}_{4}$ gas. ${ }^{11-13}$ This synthesis process yields very high purity, small $(0.1-0.3 \mu \mathrm{m})$, uniform size (geometric standard deviation typically 1.45$)$, agglomerate-free Si powders. The Si-H bonding that is characteristic of these powders is one of the important features that makes them unique. Handling and part fabrication procedures have been developed by which $\sim 65 \%$ dense, defect-free green parts can be made and dried under controlled atmosphere exposures. This powder and these capabilities have permitted properties and reaction kinetics that are substantially improved compared with prior experience.

Through this fundamental nitriding kinetic research, we have significantly reduced the severity of the nitriding schedules. ${ }^{14,15}$ If as-synthesized purity levels are maintained, complete nitridation can be accomplished in schedules 
like $1150^{\circ} \mathrm{C}$ for $1 \mathrm{~h}$ or $1250^{\circ} \mathrm{C}$ for $10 \mathrm{~m}$. The use of pre-nucleation heat treatments can reduce the required temperature to $1050^{\circ} \mathrm{C}$. Higher temperatures are required if the initial hydrogenated particle surface chemistry is altered by significant oxidation, alcohol or specific polymer exposures; however, the maximum required ternperatures are usually less than $1400^{\circ} \mathrm{C}$. These results, discussed in the next section, represent dramatic reductions from the normal schedules ( $T>$ Si melting point) which cause microstructural problems. Simply exceeding the Si melting temperature usually causes the liquid Si to flow uncontrollably in the RBSN part, where it typically collects in regions that are too large to nitride completely. Also, the volumetric expansion that occurs with solidification of Si can crack the parts. These elevated temperature levels, in combination with the liquid phase Si, aggravate interactions between phases in ceramic composites which are only marginally controlled at best.

Defect-free green microstructures, which are possible with these Si powders, have permitted RBSN strength levels to be increased to levels normally achieved only with the highest quality dense ceramics. Strengths of parts made from these powders have gradually been increased, starting from an average level of approximately $350 \mathrm{MPa}(50,000 \mathrm{psi})$ for $60-65 \%$ dense RBSN. 16,17 By using an octanol dispersant for the colloidally pressed parts, but not employing a cold isostatic pressing (CIP'ing) step, we have produced nominally $75 \%$ dense RBSN parts with a mean strength of $590 \mathrm{MPa}(85,500 \mathrm{psi})$ and a maximum of $870 \mathrm{MPa}(126,000 \mathrm{psi}) .{ }^{18}$ Strength-limiting flaws were generally 10-30 $\mu \mathrm{m}$. These results show that porosity need not have any detrimental effect on strength, provided it is uniformly distributed in pores whose dimensions are much less than the strength limiting flaws. Similar properties have recently been achieved using polymer bonded green parts made from spray dried powders. 19 If made properly, these porous, reaction-formed materials can exhibit absolute property levels that are near the maximum values exhibited by $\mathrm{Si}_{3} \mathrm{~N}_{4}$, and can have superior specific strengths (up to 0.36 $\left.\mathrm{MPa} /\left(\mathrm{kg} / \mathrm{m}^{3}\right)\right)$ and strains to failure $(\sim 0.3 \%)$ because of their low densities and reduced moduli. 20

The high strengths resulted from a combination of the anticipated small flaw size and relatively high fracture toughnesses exhibited by specific batches of RBSN samples. Contrary to predictions for this range of porosity, we have found that fracture toughness is not a simple function of density. For instance, with $75 \%$ dense RBSN samples having different processing histories and originating from different batches of Si powder, we have found $21-23$ that subtile differences in detailed microstructural features cause the $K_{I C}$ to vary from $\sim 1.6$ to $2.9 \mathrm{MPa} \cdot \mathrm{m}^{1 / 2}$. Based on maximum observed $\mathrm{K}_{I C}$ values, we concluded that $\mathrm{K}_{1 \mathrm{C}}$ decreases no faster than linearly with porosity and there is no intrinsic basis for the exponential dependence predicted by popular models. For largely undefined reasons, RBSN nitrided in a high pressure $\mathrm{N}_{2}$ atmosphere, 500 atm $)^{24}$ exhibited slightly different microstructural features and a toughness of 
$5.4 \mathrm{MPa} \cdot \mathrm{m}^{1 / 2}$. Although not fully understood, these results demonstrate the important effect synthesis reaction mechanisms have on the properties of the monolithic reaction formed ceramics through direct effects on resulting microstructures. The effects can be even more dramatic when RBSN is used as a matrix for ceramic matrix composites. Using large diameter SiC fiber reinforcements Bhatt $25-28$ demonsirated that ultimate flexural surface stress at failure ( $3 \& 4$ point loading) was increased up to 9.5 times, the flexural stress at which the matrix first cracked were increased up to 2.7 times, and the failures were not brittle even in tension. For similar RBSN matrix composites, Corbin29-31 demonstrated work of fracture values for these composites that were improved 10-20 times relative to monolithic RBSN.

Oxidizing exposures have two principal damaging effects on RBSN. If oxidation occurs within the parts, creep rates become comparable to SSN or HPSN, so maximum service temperatures are limited to about $1200^{\circ} \mathrm{C}$. If the oxide layer becomes too thick when it becomes protective, a polymorphic cristobalite transformation at about $250^{\circ} \mathrm{C}$ causes cracks originating in the oxide film to propagate into the RBSN body; these cracks become the strength limiting flaws.

Oxidation kinetics of the high purity RBSN have been measured at 1000 and $1400^{\circ} \mathrm{C}$, as well as room temperature strengths after 1 and $50 \mathrm{~h}$ exposures at these temperatures. ${ }^{32}$ With the high purities and very fine pore structures that characterize these RBSN parts, a thin protective layer forms very quickly. Total weight gains per unit area $\left(\sim 10^{-9}-10^{-7} \mathrm{~g} / \mathrm{cm}^{2} / \mathrm{s}\right.$ at 1000 and $\left.1400^{\circ} \mathrm{C}\right)$ are $10-$ 20 times less than is observed either with high quality RBSN or fully dense hot pressed $\mathrm{Si}_{3} \mathrm{~N}_{4}$ (HPSN) at the same temperature levels. For the cases studied, the exterior protective oxide layers were only 1-2 $\mu \mathrm{m}$ thick, so widely distributed cracks did not have adequate strain energy to propagate into the RBSN substrates. Unlike other RBSN's ${ }^{33,34}$ and HPSN's ${ }^{35}$ subjected to the same oxidizing exposures, these parts did not exhibit any decrease in strength after ( xidation. The high purities and fine pore structures, generally accessible to $r t$ action formed ceramics, were responsible for these differences. Based on independently measured high temperature strengths of RBSN in nonoxidizing atmospheres, 33,34 these oxidation characteristics should make possible retention of strengths and creep resistance to the dissociation temperature $1800^{\circ} \mathrm{C}$ in oxidizing atmospheres.

Minimal dimensional change during the consolidation process is another important feature of this reaction formed material. From die to fired part, the total dimensional change for most RBSN parts is in the range of $0.5-2.5 \%$ with a standard deviation of a few tenths of a percent. Methanol-processed, CIP'ed, $\mathrm{SiH}_{4}$-derived RBSN exhibits a total dimensional change of $1.9 \pm 0.26 \%$ while the octanol processed RBSN exhibits a total change of only $0.2 \pm 0.25 \% .18$ While impressive when compared to shrinkages observed with conventional densification processes (10-20\%), filled RBSN samples allow even tighter tolerances to be maintained. For parts made from a mixture of $88 \%$ Si and $12 \%$ 
$\mathrm{SiC} \mathrm{SiH}_{4}$ derived powders, the total dimensional change was $0.1 \pm 0.05 \% .18$ (A 1-inch die would produce a part that is $0.999 \pm 0.0005$ inches). This impressive dimensional stability and variance permitted Hitachi Ltd. 36 to make ceramic gears and bearings based on RBSN/SiC composites that do not include any grinding operations. While the cost-savings of a net-shape fabrication process is the most evident consequence of achieving these dimensional tolerances, eliminating the uncontrolled flaw population that results from grinding will probably prove to have greater importance.

The dielectric constants and loss tangents of MIT RBSN samples have been measured at $35 \mathrm{GHz}$ for $\mathrm{T} \leq 1200^{\circ} \mathrm{C}$. In contrast to SSN and HPSN, losses from ionic transport in high purity RBSN should be minimal because the grain boundaries are free of glass forming impurities. Nonintrinsic dielectric losses should result primarily from trace levels of residual Si. Experimental results confirm anticipated behavior. Samples containing less than $0.1 \%$ residual $\mathrm{Si}$ exhibited loss tangents as low as $10^{-5}$ at low temperatures, and $<10^{-3}$ for temperatures up to $1200^{\circ} \mathrm{C} .19$ Again, the porosity that is characteristic of RBSN proves advantageous by lowering dielectric constants to a range between 4.8 and 5.2. Demonstrated dielectic, thermal and mechanical properties make the high purity RBSN an attractive choice for many substrate and window applications. With improved nitriding procedures, loss tangents and the temperature coefficient values that are more similar to those of CVD $\mathrm{Si}_{3} \mathrm{~N}_{4}$ should be achievable, permitting RBSN microwave windows to be operated at temperatures up to the dissociation temperature of $\mathrm{Si}_{3} \mathrm{~N}_{4}\left(\sim 1800^{\circ} \mathrm{C}\right)$.

These results illustrate that reaction forming processes can overcome longstanding deficiencies if synthesis is carried out properly. Several important parts based on chemical vapor phase infiltration $(C V I)$, reaction bonded silicon carbide (RBSC), reaction bonded aluminum oxide (RBAO), direct oxidation and nitridation (DIMOX ${ }^{\mathrm{TM}}$ ), self propagating high temperature reactions (SHS) and polymer derived ceramics, are finding commercial application because of the unique combination of processing advantages and properties. Controlling the reaction mechanisms is the key to achieving critical performance thresholds because most of the normally encountered microstructural defects are directly attributable to processing issues. Although RBSN based ceramics have great imporiance in their own right, they may ultimately prove more significant as a model for other reaction formed ceramic materials.

\section{Nitriding Kinetics of High PuRITY Silicon POWders}

\section{A. Materials and Experimental Procedures}

Nitriding kinetics were studied ${ }^{37}$ with $\mathrm{Si}$ powders synthesized from $\mathrm{CO}_{2}$ laser heated $\mathrm{SiH}_{4}$ gas using procedures that have been reported. ${ }^{11-13}$ Most of the nitriding research was done with a limited number of batches of powder produced under identical conditions. This standard powder had a B.E.T. single point surface area of $10.5 \pm 1.0 \mathrm{~m}^{2} / \mathrm{g}$. TEM-determined particle size 
distributions showed the powders to have log-normal distributions, a mean (mass) diameter of $0.305 \mu \mathrm{m}$, and a geometric standard deviation of 1.5 .

Smaller powders, having a BET surface area of $18.5 \pm 1.0 \mathrm{~m}^{2} / \mathrm{g}$, were used to study the effect of particle size. TEM analyses showed that the particles were free of agglomerates, a conclusion confirmed by the packing densities of both dry pressed (51-54\%) and colloidally pressed (63-68\%) samples. FTIR spectra of the as-synthesized powders showed a dominant feature of two closely spaced absorption bands at 2080 and $2094 \mathrm{~cm}^{-1}$, respectively corresponding to $\mathrm{Si}-\mathrm{H}$ and $\mathrm{Si}-\mathrm{H}_{2}$ bonding on the particle surfaces. Fresh powder spectra show a weak $\mathrm{Si}-\mathrm{O}$ absorption band at $1100 \mathrm{~cm}^{-1}$.

The oxygen content of as-synthesized and air-exposed powders were measured by neutron activation to determine the consequences of air exposures during sample fabrication. The results are summarized in Table 1. These results show that the powder is remarkably free of $\mathrm{O}_{2}$ contamination as synthesized, and oxidizes very slowly at room temperature in air. The brief exposure to air encountered during transferral of the pellets into the nitriding furnace should cause the $\mathrm{O}_{2}$ content to increase by less that $2 \%$ of a monolayer. The glove box atmosphere retains the powders' purity for essentially indefinite periods.

Nitriding samples were either pressed pellets or loose powders. Both drypressed and colloidally-pressed pellets $(1.3 \mathrm{~cm}$ diameter, 1-5 mm thick) were studied. Double pressing (press at $34 \mathrm{MPa}$, grind, press at $67 \mathrm{MPa}$ ) was used for the dry-pressed samples to raise the packing density slightly $(\sim 51-54 \%)$ and to give them adequate handling strength. The colloidal pressing technique has been reported extensively. ${ }^{16-23}$

Table 1. Oxygen Content of As-Synthesized and Air-Exposed Si Powders

$\begin{array}{ccc}\begin{array}{c}\text { Powder } \\ \text { Exposure }\end{array} & \begin{array}{c}\text { Measured } \mathrm{O}_{2} \\ \text { (opm) }\end{array} & \begin{array}{c}\text { Fractional Coverage } \\ \text { (\% Monolaver) }\end{array} \\ \text { As Synthesized } & 162 & 3.8 \\ \text { Air: } 1 \mathrm{hr} & 266 & 6.2 \\ 2 \mathrm{hrs} & 364 & 8.5 \\ 1 \text { day } & 601 & 14.0 \\ 60 \text { days } & 3010 & 64.0\end{array}$

Glove $\operatorname{Box}\left(\mathrm{N}_{2} \mathrm{~atm}\right)$ :

60 days $\quad 215$

Most of the samples were nitrided in a Netzsch STA-429 thermogravimetric analysis (TGA) apparatus that had been modified to insure atmospheric control. These modifications included installation of a vacuum capability, cold trap, 
copper mesh getter and precise gas flow meters, replacement of plastic plumbing with metal, and attachment of an oxygen measuring device. Ultrahigh-purity $\mathrm{N}_{2}$ (combined $\mathrm{O}_{2}$ and $\mathrm{H}_{2} \mathrm{O} \leq 10 \mathrm{ppm}$ ) was used for the nitriding experiments; $\mathrm{O}_{2}$ contents of entering and exiting gases were $\leq 1 \mathrm{ppm}$. Some samples were nitrided in a Centorr tungsten mesh furnace located within the $\mathrm{N}_{2}$ atm. glove box to minimize $\mathrm{O}_{2}$ contamination. All samples were contained in high purity RBSN fixtures (powders in cups, pellets vertically in a boat). In general, samples were heated rapidly to the nitriding temperatures in $\mathrm{N}_{2}$, and then held isothermally; preheating in an inert atmosphere caused partial densification and some coarsening.

\section{B. Macroscopic Nitriding Kinetics}

Typical TGA results for dry-pressed Si powders are shown in Figures 1a and $1 \mathrm{~b}$ for temperatures ranging from 1050 to $1350^{\circ} \mathrm{C}$. The two figures partially represent the same set of data over different time scales. The $1250^{\circ} \mathrm{C}$ data show $>97 \%$ conversion in less than 10 minutes. The curves exhibit three distinct periods:

1. An initial induction period where there is little nitridation,

2. A "fast" reaction period, in which most of the nitridation occurs,

3. A "slow" reaction period following the fast period, in which nitriding continues at a rate that decreases asymptotically to zero.

Experiments were undertaken to understand the mechanisms responsible for each of these characteristic periods.

The phase content at the end of the fast reaction periods as a function of distance from the exposed surfaces for dry-pressed samples is shown in Figures $2 a$ and $2 b$. These $X$-ray diffraction results reveal an unusual reverse reaction gradient, in which the interiors of the samples nitride more extensively than the exterior regions. The evolution of these gradients is shown for 1150 and $1250^{\circ} \mathrm{C}$ samples at various intervals during the fast reaction period.

The results show that complete nitridation occurred locally in as little as 45 minutes at $1150^{\circ} \mathrm{C}$, and that some combination of transport-processes or mechanisms act to either inhibit the reactions near the exterior surfaces of the samples or to accelerate the reactions within them. Possible sources of the reverse gradients are:

1. Temperature gradients caused by the exothermic reaction,

2. Convective cooling of the outer surfaces,

3. Nitrogen gradients through the pore structure,

4. Silicon vapor gradients through the pore structure,

5. Residual oxygen or water in the nitriding gas which reacts with either solid surfaces or the reactant gases near the outer surfaces. 

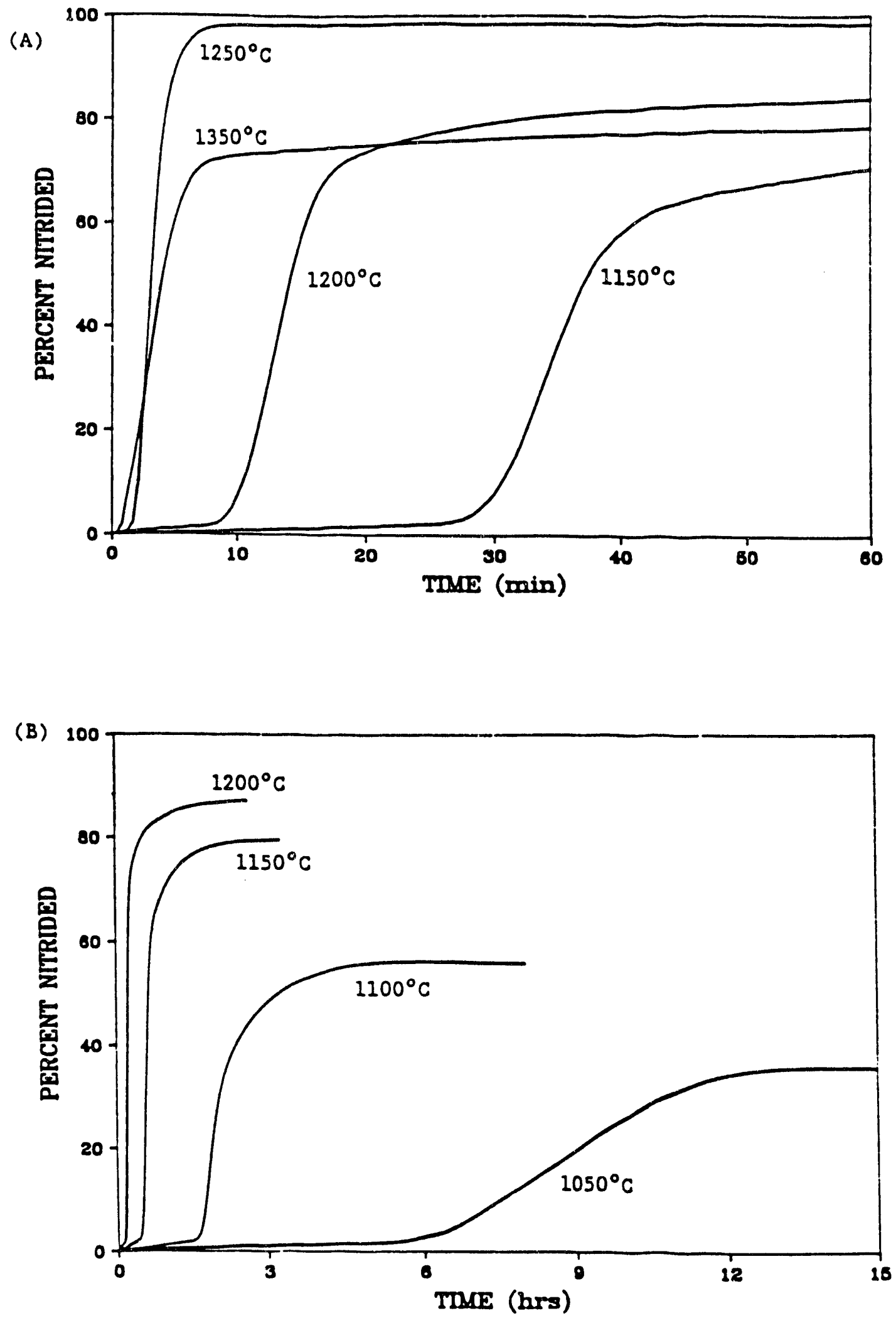

Figure 1. Isothermal TGA data: (A) First hour $\quad$ (B) Long times 

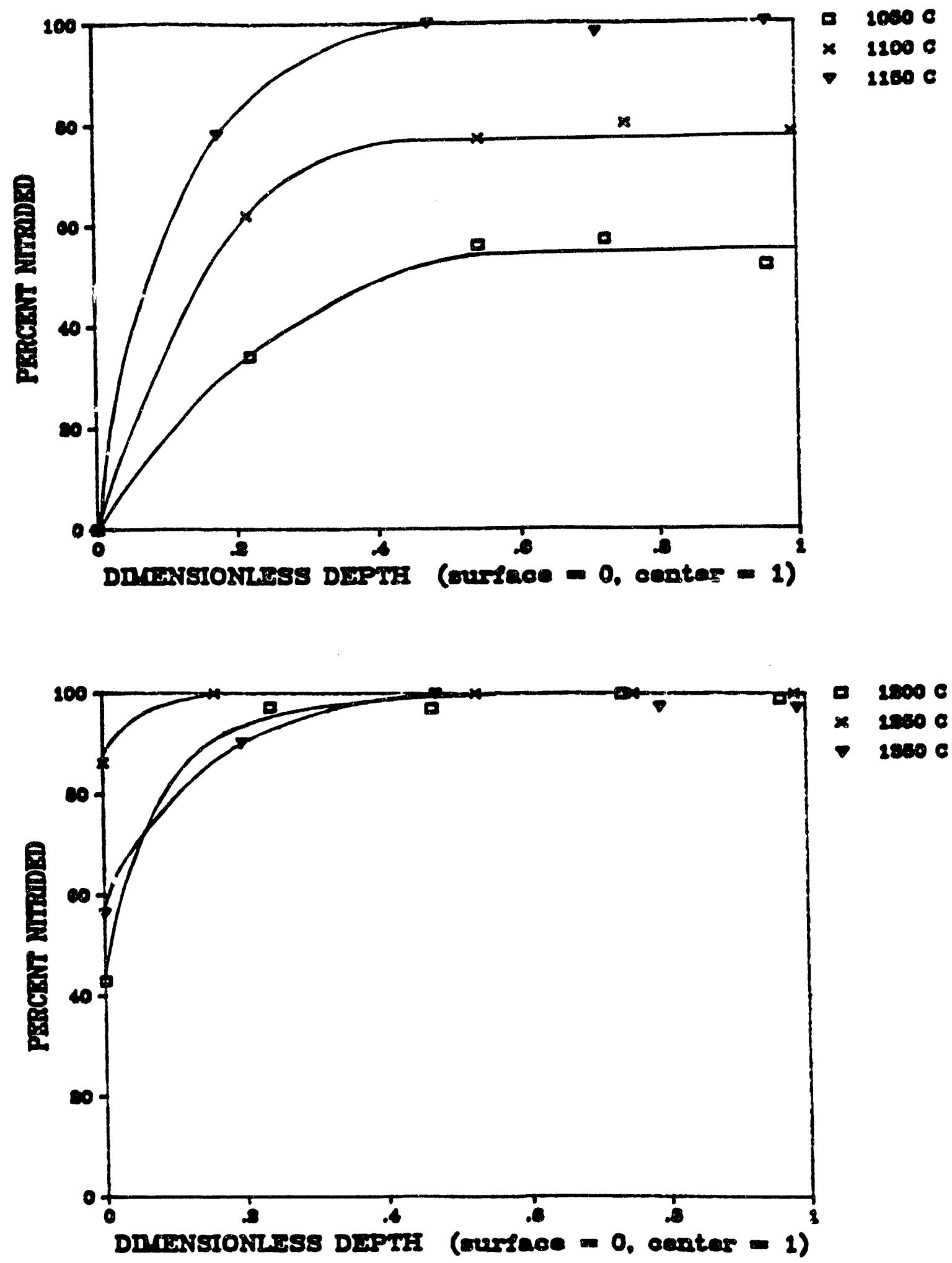

Figure 2. Composition gradients determined by quantitative $X$-ray diffaction, for samples that were isothermally nitrided through the end of the "fast" reaction. 
Experiments and analyses were undertaken to determine which of these possible effects may be responsible for the gradients that appeared to result from a local inhibition of the nitridation reactions. These results will be abstracted.

Nitriding experiments were undertaken with varied sample thicknesses and $\mathrm{N}_{2}$ flow rates to isolate the effects of heat and mass transport both within the porous samples and through the external boundary layers. With the exception of the $1350^{\circ}$ samples, increased sample thickness led to both increased overall- and surface-reactions. Increased gas flow rate had no effect on the extent of the reactions near sample centers, but it did progressively suppress the extent of reaction near the samples' exterior surfaces.

Samples were partially oxidized by exposure to air at $200^{\circ} \mathrm{C}$ in the TGA to investigate the effects of submonolayer concentrations of $\mathrm{O}_{2}$ on the nitriding kinetics (on the standard Si powders, a weight gain of $0.51 \%$ corresponds to 1 monolayer of oxygen). Samples with 30,60 and $90 \%$ of a monolayer were nitrided isothermally at temperatures ranging from 1200 to $1350^{\circ} \mathrm{C}$. The $90 \%$ samples all underwent partial densification during nitriding, so the effects of oxidation and densification cannot be isolated. For the other samples, even these small oxygen concentrations led to extended induction times and to less extensive conversion during the fast reaction periods. Compositional gradients in these nitrided samples were similar to those exhibited by the unoxidized samples, but had somewhat lower conversions to $\mathrm{Si}_{3} \mathrm{~N}_{4}$.

Extensive heat transfer analyses were undertaken to determine whether the reaction gradients resulted from either the exothermic reactions or convective cooling by the flowing $\mathrm{N}_{2}$ gas. These results showed that radiation to the surroundings rather than convection through the boundary layer was the principal source of heat transfer. The calculations indicated that the sample temperatures increased above the furnace temperatures during the fast reaction periods $\left(\sim 2^{\circ} \mathrm{C}\right.$ for $1050^{\circ} \mathrm{C}$ samples, up to $\sim 110^{\circ} \mathrm{C}$ for thick samples nitrided at $1350^{\circ} \mathrm{C}$ ); however, the maximum temperature differential within the samples was always less than $11^{\circ} \mathrm{C}$. Although melting of the residual Si was predicted for the $1350^{\circ} \mathrm{C}$ sample, nitrided samples exhibited no microstructural evidence of liquid Si. Heat transfer was eliminated as a probable dominant cause for the reverse gradients because the predicted temperature differentials were very low, and because comparable or higher local temperatures near exterior surfaces did not cause as extensive nitridation as occurred internally.

Nitrogen gradients within the samples might be responsible for the reverse reaction gradients if, for instance, high $\mathrm{N}_{2}$ concentrations enhanced nucleation to the extent that free Si surfaces were prematurely blocked off. Although these analyses show that significant $\mathrm{N}_{2}$ gradients probably exist within the thicker, higher-temperature samples during the fast reaction periods, it is unlikely that $\mathrm{N}_{2}$ transport has any significant effect on the overall reaction kinetics because 
the reaction rates are the same for all sample thicknesses. Also, the extents of reaction were unchanged at the centers of varied thickness samples where the maximum consequence of varied gradients should be observed.

Two limiting cases were examined for Si vapor phase transport to determine whether this mechanism could be responsible for the reverse gradients. In the first, it was assumed that Si vapor traveled to the exterior without reacting to form RBSN. In the second, it was assumed that a "distributed sink" corresponding to the nitridation reaction competed with the outward diffusive transport. The first corresponds to the induction period in which little or no reaction occurs; the second corresponds to the fast reaction period. Various limiting boundary layer assumptions were made.

Qualitatively, Si vapor gradients can explain the observed composition gradients if a lower $\mathrm{Si}$ concentration near the outer surfaces produces less conversion to $\mathrm{Si}_{3} \mathrm{~N}_{4}$. The observed $\mathrm{N}_{2}$ gas flow rate effects are consistent with $\mathrm{Si}$ vapor gradients if $\mathrm{Si}$ transport occurs in a mixed control regime where diffusion through the pore structure and the boundary layer are both partially rate-controlling. The observation that the conversion at the outer surface increases slightly or not at all with increasing thickness is also consistent with $\mathrm{Si}$ vapor transport being responsible for the gradients.

The analyses show that for the first case, where there is no sink and when a diffusional boundary layer exists, the $P_{S i}$ is uniform throughout the part; for the nonreacting case, gradients exist only if the porous part is heated in a vacuum. In contrast, significant differences can exist in $\mathrm{P}_{\mathrm{Si}}$ throughout the fast reaction period and the predicted gradients increase as the Si particle surface area decreases with partial coverage by $\mathrm{Si}_{3} \mathrm{~N}_{4}$ reaction product. These calculations and weight-loss rate experiments in vacuum also show that the overall rate controlling step for the nitriding process is very likely to be Si vaporization.

The role of oxygen contamination was deduced qualitatively. If all of the residual oxygen $(<1 \mathrm{ppm})$ in the gettered $\mathrm{N}_{2}$ gas was captured uniformly by the Si pellets, the contamination would be negligible except at the highest flow rates studied. However, if the oxygen were concentrated at the surfaces of the pellets, then oxygen contaminations $>10 \%$ of a monolayer would result locally even at the slowest flow rates. Based on the controlled oxidation experiments, these levels are high enough to significantly retard nitriding kinetics near the surfaces. Also, samples exposed for longer periods at temperature levels where oxidation is rapid, $\mathrm{T}>150^{\circ} \mathrm{C}$, but at which nitridation does not occur, $\mathrm{T}<700^{\circ} \mathrm{C}$, exhibited significantly retarded nitriding kinetics. The most convincing evidence that oxygen contamination was responsible was that the presumed oxidation effects acted to stop or retard nitriding by extending the induction times at the surfaces without affecting the rates near the sample centers. Other transport phenomena should have resulted in nitridation reactions beginning at the same time throughout the samples, but at different 
rates. This combination of observations led us to conclude that localized oxidation of the outer surfaces is the primary cause of the observed composition gradients. Although the results point to residual oxygen as the cause of the effect, the process may be indirect; for example, the oxygen may react with the $\mathrm{Si}$ vapor to form $\mathrm{SiO}$, or potentially with the $\mathrm{N}_{2}$ gas to form much less favored NO.

Three sets of experiments were undertaken to further reduce the $\mathrm{O}_{2}$ contamination levels in the $\mathrm{N}_{2}$ gas stream. Two of these consisted of nitriding within the W-mesh furnace without any air exposure with the samples packed in other Si pellet fragments, and within the TGA apparatus with the samples packed in a Si powder bed; the excess, high surface area Si was intended to getter residual $\mathrm{O}_{2}$ before it reached the samples. In both cases, complete, uniform nitridation was achieved at $1150^{\circ} \mathrm{C}$, as was the case for the interior regions of samples that were fired conventionally. The $1250^{\circ} \mathrm{C}$ sample also nitrided to completion in the $W$-mesh furnace, while the sample packed in powder overheated, causing the Si to melt. The third set employed forming gas $\left(95 \% \mathrm{~N}_{2}-5 \% \mathrm{H}_{2}\right)$ to further lower the $\mathrm{PO}_{2}$. The nitriding kinetics for these samples exhibited no change within interior regions, and slightly shortened induction times and similar fast reaction rates at the exterior regions of the samples. The effect of the forming gas was minimal, but it might be made more effective by active drying to remove residual water.

The macroscopic nitriding kinetic studies have shown that complete nitridation can be achieved at temperatures less than $1150^{\circ} \mathrm{C}$ in times less than 1 hour. Both time and temperature levels are substantially lower than is true for conventional $\mathrm{Si}$ materials. The results have also shown that achieving these rapid rates depends critically on maintaining high purity levels. Although care is necessary, the measures needed to assure complete reaction are manageable, and represent important options for making ceramic matrix composites.

\section{Nitrogen Adsorption on Silicon Powder Surfaces}

Nitridation begins with the formation of $\mathrm{Si}-\mathrm{N}$ bonds on the Si powder surfaces. Changes in the initial powder surface chemistry were studied as a prerequisite to studying $\mathrm{Si}_{3} \mathrm{~N}_{4}$ nucleation and growth.

The nitrogen adsorption process was studied by FTIR and both dynamic (heating rate $2-20^{\circ} \mathrm{C} / \mathrm{min}$ ) and isothermal (heating rate $50^{\circ} \mathrm{C} / \mathrm{min}$ to $\mathrm{T}_{\text {hold }}$ ) TGA characterization techniques; loose powders and dry-pressed pellets were fired in a static $\mathrm{N}_{2}$ atmosphere. A static atmosphere was employed to reduce the contamination level and to minimize the TGA noise level since the mass change is very small (1-3 mg) even with relatively large samples $(500-800 \mathrm{mg})$. Loose powders were used as FTIR samples.

TGA and FTIR results show that a monolayer of nitrogen replaces the hydrogen on the surfaces of the powder at temperatures between 700 and $950^{\circ} \mathrm{C}$, 
followed by a continued slow weight gain as more chemisorbed nitrogen attaches to the particles. FTIR spectra of as-synthesized samples that were heated rapidly to $800^{\circ} \mathrm{C}$ and then held in $\mathrm{N}_{2}$ for periods of 1 and 3 hours are shown in Figure 3 . These results show that the Si-N bonds that form (830-860 $\mathrm{cm}^{-1}$ ) resemble those in amorphous $\mathrm{Si}_{3} \mathrm{~N}_{4} ; 38$ the absorption doublet at 2100 $\mathrm{cm}^{-1}$ in this figure shows that some hydrogen remains bonded to the surface after one hour, but it is completely displaced by nitrogen after 3 hours. FTIR spectra of both normally processed samples and samples that were previously oxidized to produce 30 and $60 \%$ of a surface monolayer of oxygen coverage, show that the $\mathrm{Si}-\mathrm{O}$ bonds remain intact while $\mathrm{Si}-\mathrm{N}$ bonds form. The nitriding reactions in the $700-950^{\circ} \mathrm{C}$ range are similar for oxidized and as-synthesized powders, except that the presence of $\mathrm{Si}-\mathrm{O}$ bonds reduces the amount of the $\mathrm{Si}$ surface that is available for nitrogen chemisorption. The results indicate that the hydrogen must be desorbed befcre the nitrogen can be adsorbed; however, it has not been determined whether the two steps occur simultaneously.

Isothermal TGA results for as-synthesized Si powders are shown in Figure 4. The concave-convex shapes of these curves indicates that the process depicted in Figure 4 does not correspond to a simple 1-step mechanism. To test whether desorption of hydrogen was responsible for the multistep behavior, samples were heated for 30 minutes in vacuum at $900^{\circ} \mathrm{C}$ prior to nitridation. Isothermal TGA results for the vacuum treated powders, shown in Figure 5, exhibit only negative curvature. FTIR spectra of vacuum treated powders shows that all of the hydrogen is removed from the Si powder surfaces for temperatures in excess of $600^{\circ} \mathrm{C}$.

Adsorption kinetics for vacuum treated Si powders were analyzed to give the order of the reaction, the activation energy and the sticking probability. Regression analyses showed the best fit for first order kinetics, an activation energy of $142 \mathrm{~kJ} / \mathrm{mole}$, and an extremely low sticking probability between $2.5 \mathrm{x}$ $10^{-12}$ and $5.2 \times 10^{-11}$. It was further deduced that the absorbing species is probably $\mathrm{N}_{2}$ and that 2 adjacent surface sites are not required.

The vacuum treated samples showed extreme sensitivity to spontaneous oxidation after the bonded hydrogen had been removed from the assynthesized surfaces. This observation has important consequences for specifying binder-burnout schedules and subsequent exposures.

\section{D. $\mathrm{Si}_{3} \mathrm{~N}_{4}$ Nucleation and Growth}

Samples were analyzed by TEM, X-ray diffraction, and FTIR throughout the nitriding process to provide descriptions of the nucleation and growth steps. Most nitriding was carried out at 1150 and $1250^{\circ} \mathrm{C}$ so that samples could be removed for characterization at predictable levels of conversion. The onset of the fast reaction proceeded so rapidly at higher temperatures that it was 


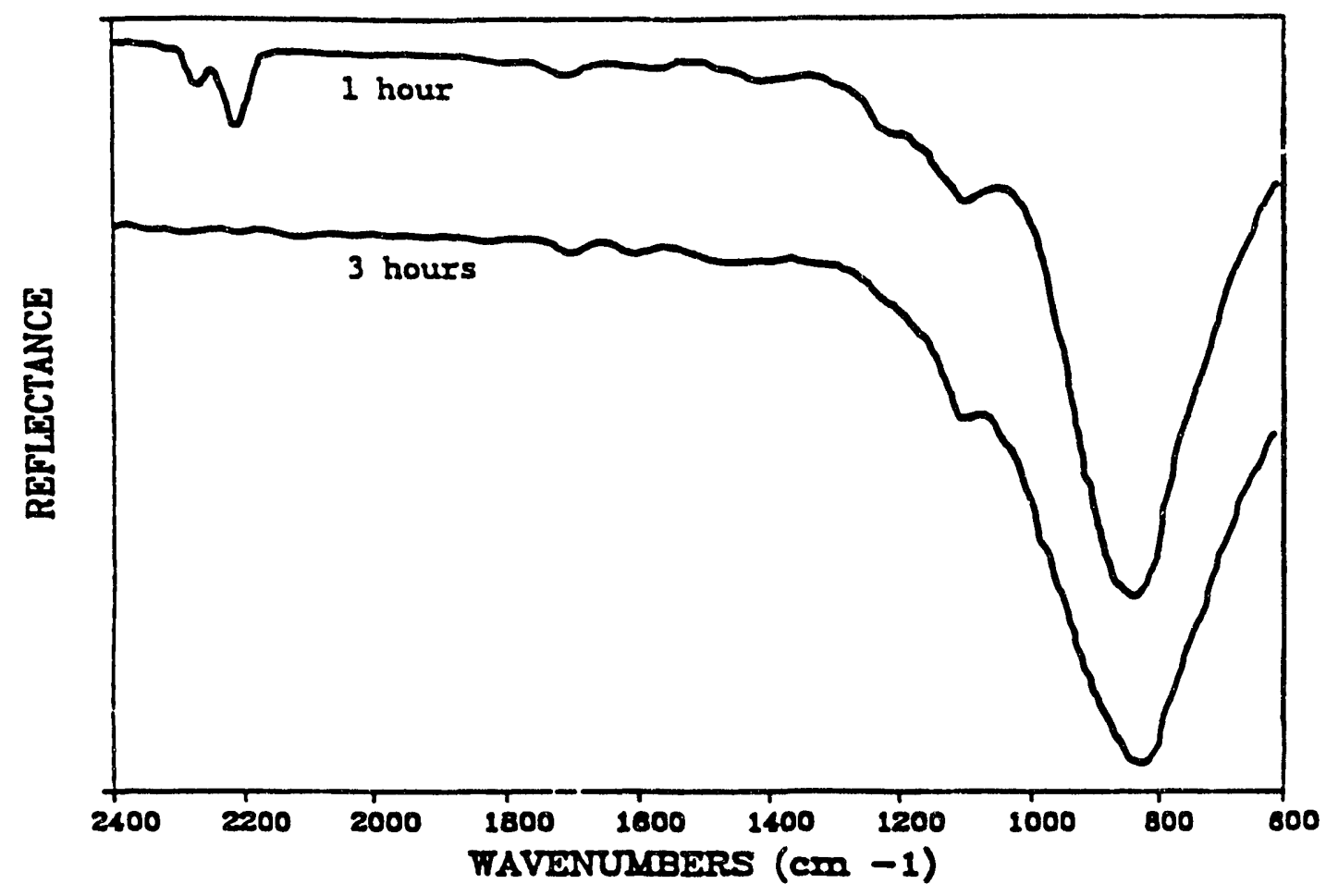

Figure 3. FTIfi spectra: loose powder fired at $800^{\circ} \mathrm{C}$ for one hour and three hours.

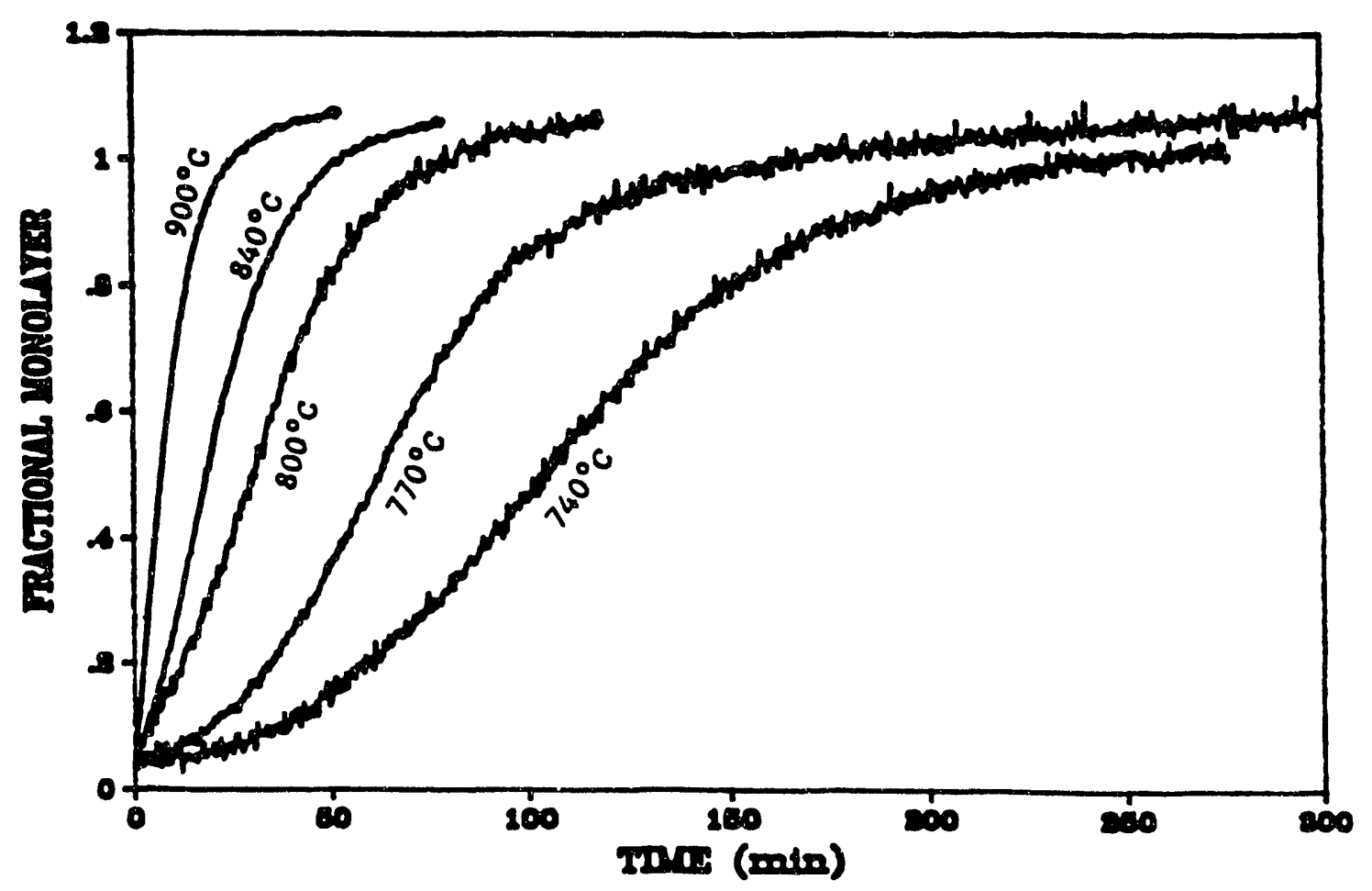

Figure 4. Isothermal data, samples fired in nitrogen. 


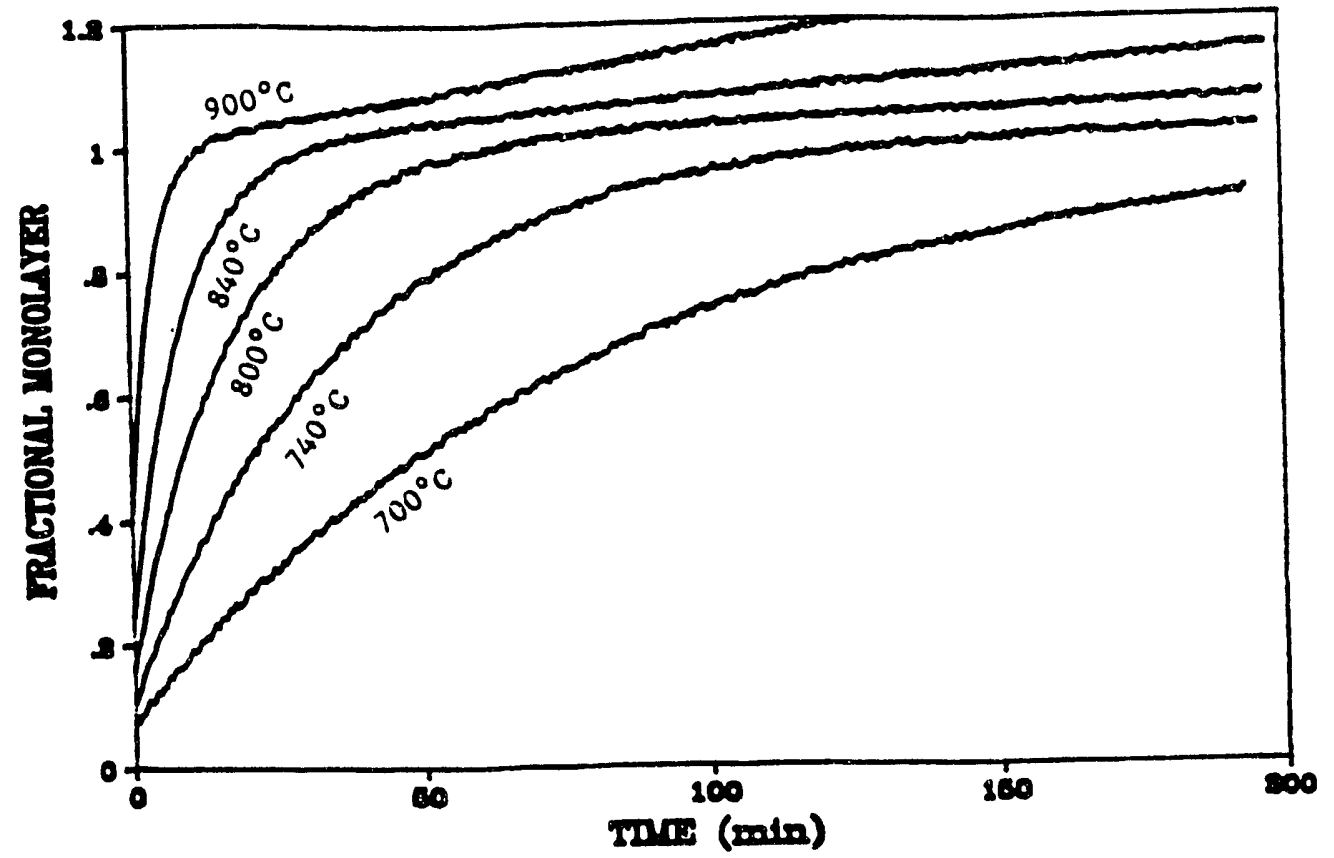

Figure 5. l'sothermal data: samples fired under vacuum for one hour at $900^{\circ} \mathrm{C}$, and then fired at the temperature shown in $\mathrm{N}_{2}$.

impossible to interrupt the process reliably. The samples that were investigated are summarized on Figure 6 and in Table 2. Figure 6 plots the initial TGA weight-gain data for an isothermal $1150^{\circ} \mathrm{C}$ sample, in which the numbers $1-5$ correspond to the identical numbers in Table 2 to illustrate how the firing times for these samples correspond to the progression of the induction and fast resction periods.

FTIR spectra for samples 1 and 3 are shown in Figure 7. These spectra, which are nearly identical to the other two induction-period samples 2 and 8 , exhibit the broad $830 \mathrm{~cm}^{-1}$ band corresponding to amorphous $\mathrm{Si}-\mathrm{N}$ bonds and a weak $1100 \mathrm{~cm}^{-1}$ peak corresponding to small levels of oxygen contamination. These results show that the character of the amorphous silicon nitride layer does not change appreciably during the induction period after its initial formation by chemisorption processes. TGA results indicate that the amorphous layer is approximately $12 \AA$ thick at the end of the induction period. In contrast, samples taken from the fast reaction period (samples 4 and 7 ) exhibit a single absorption band at $685 \mathrm{~cm}^{-1}$ and a series of three bands between 850 and $1050 \mathrm{~cm}^{-1}$; all four of these are fundamental bands for crystalline $\mathrm{Si}_{3} \mathrm{~N}_{4} .{ }^{38}$ The spectra change fundamentally with the small change in exposure that coincides with the initiation of the fast reaction period. No spectra exhibited both amorphous and crystalline peaks. Also, no evidence of $\mathrm{Si}-\mathrm{H}$ bonding was observed in samples heated to these temperature levels.

$X$-ray diffraction analyses of the samples revealed crystalline silicon nitride peaks only for samples taken beyond the induction period. Quantitative 


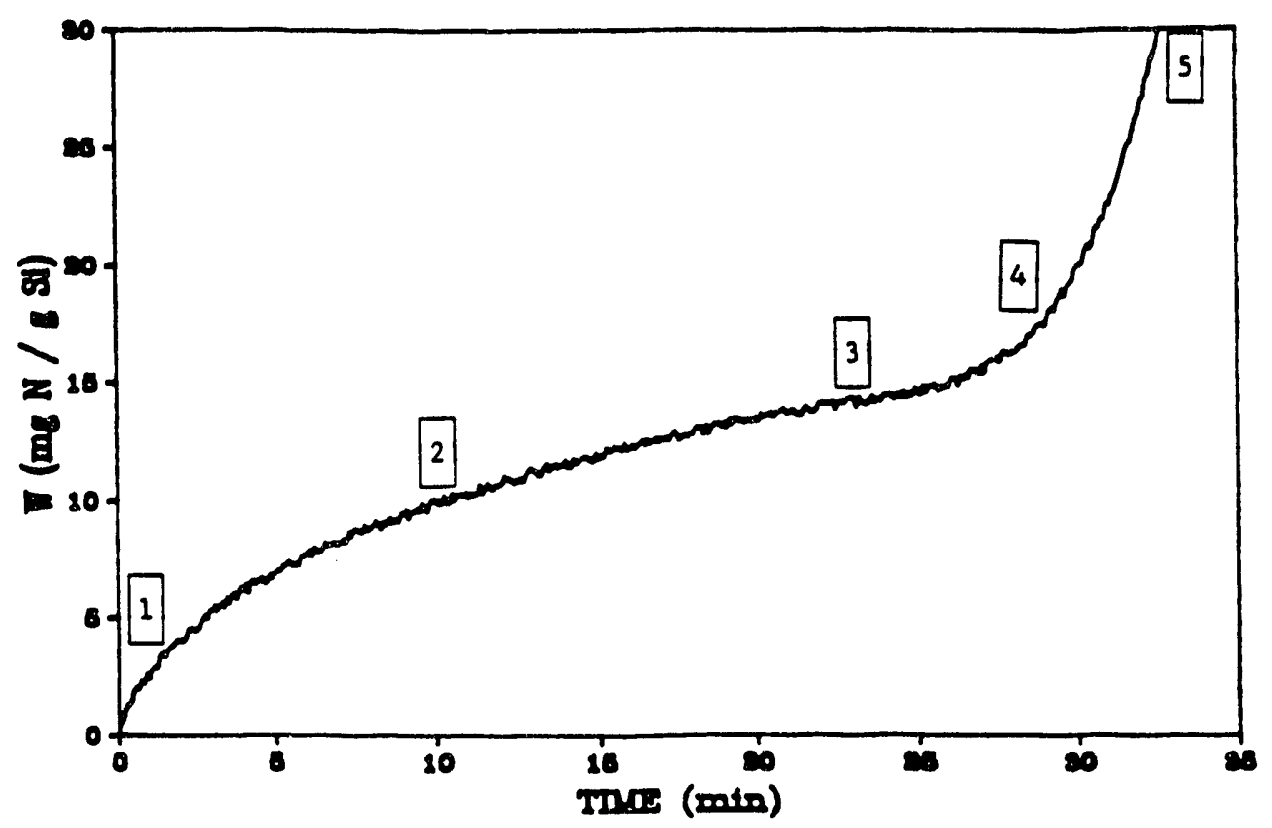

Figure 6. Initial TGA data for samples fired at $1150^{\circ} \mathrm{C}$. The numbers 1 through 5 correspond to the sample numbers in Table 2.

Table 2. Nitriding History of Powder Samples used for FTIR and TEM

\begin{tabular}{|c|c|c|}
\hline $\begin{array}{l}\text { Sample } \\
\text { Number }\end{array}$ & $\begin{array}{c}\text { Firing Temp* } \\
\text { /Time }\end{array}$ & Wt Gain"* \\
\hline 1 & $1150^{\circ} \mathrm{C} / 1 \mathrm{~min}$ & $<1 \%$ \\
\hline 2 & $1150^{\circ} \mathrm{C} / 10 \mathrm{~min}$ & $<1 \%$ \\
\hline 3 & $1150^{\circ} \mathrm{C} / 23 \mathrm{~min}$ & $1 \%$ \\
\hline 4 & $1150^{\circ} \mathrm{C} / 28 \mathrm{~min}$ & $4 \%$ \\
\hline 5 & $1150^{\circ} \mathrm{C} / 33 \mathrm{~min}$ & $13 \%$ \\
\hline 6 & $1150^{\circ} \mathrm{C} / 36 \mathrm{~min}$ & $27 \%$ \\
\hline 7 & $1150^{\circ} \mathrm{C} / 45 \mathrm{~min}$ & $43 \%$ \\
\hline 8 & $1250^{\circ} \mathrm{C} / 0 \mathrm{~min}$ & $1 \%$ \\
\hline 9 & $1250^{\circ} \mathrm{C} / 4 \mathrm{~min}$ & $16 \%$ \\
\hline 10 & $1250^{\circ} \mathrm{C} / 6 \mathrm{~min}$ & $30 \%$ \\
\hline 11 & $1250^{\circ} \mathrm{C} / 9 \min$ & $49 \%$ \\
\hline
\end{tabular}

Description
beginning of the induction period
middle of the induction period
shortly before "fast" rxn onset
shortly after "fast" rxn onset
early in the "fast" reaction
midway in the "fast" reaction
end of the "fast" reaction
shortly before "fast" rxn onset
early in the "fast" reaction
middle of the "fast" reaction
end of the "fast" reaction

* the time of zero minutes corresponds to the beginning of the induction time ** relative to the initial sample weight.

analyses ${ }^{39}$ showed that the $\mathrm{Si}_{3} \mathrm{~N}_{4}$ was $65-85 \%$ alpha throughout the nitriding process and that the percentage of $\mathrm{Si}_{3} \mathrm{~N}_{4}$ agreed with values determined by weight gain.

Previous researchers ${ }^{1}$ have suggested that $\alpha-\mathrm{Si}_{3} \mathrm{~N}_{4}$ forms by a vapor phase reaction above the $\mathrm{Si}_{3} \mathrm{~N}_{4}$ growth interface and $\beta-\mathrm{Si}_{3} \mathrm{~N}_{4}$ forms by nitrogen reacting with a condensed phase, either solid $\mathrm{Si}_{3} \mathrm{~N}_{4}$ or a liquid phase flux. 


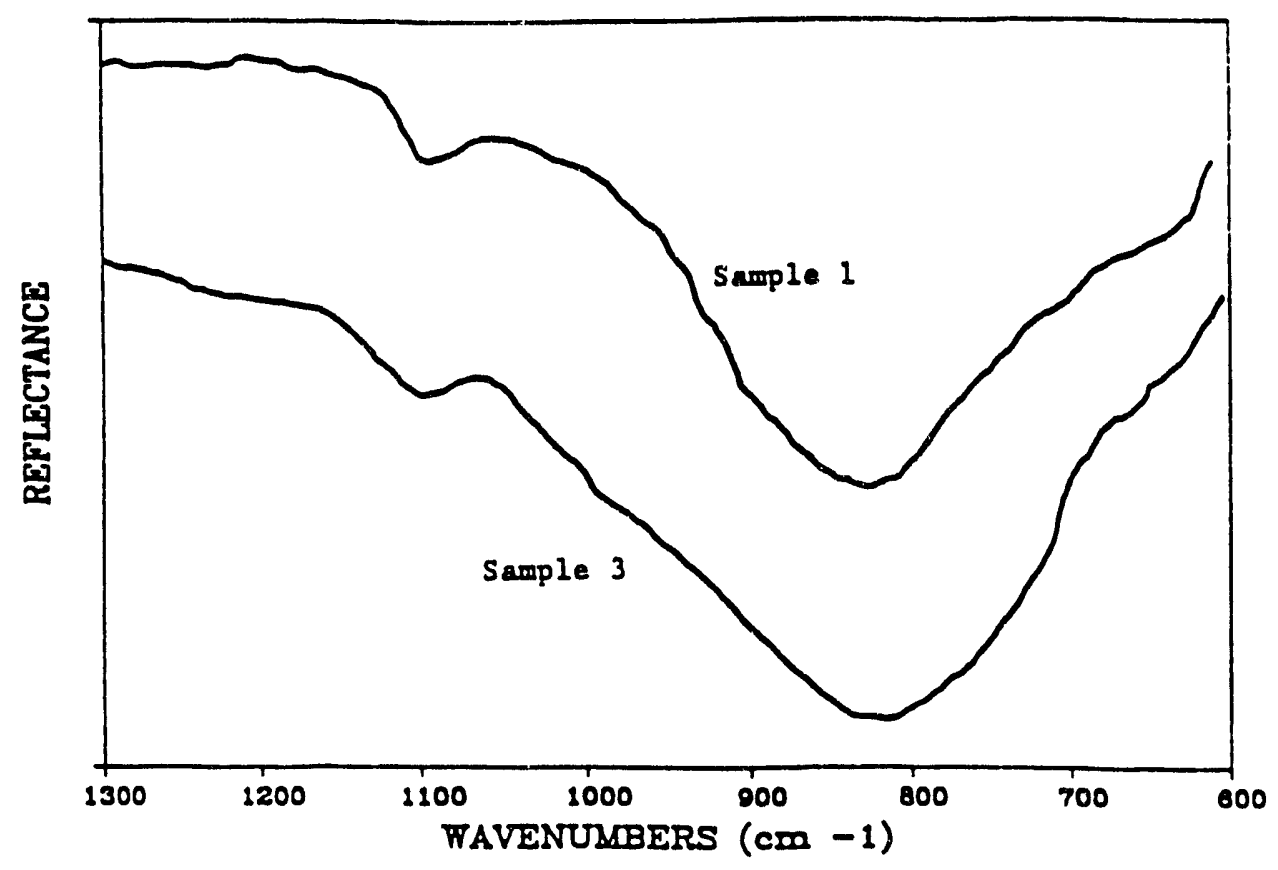

Figure 7. Induction Period FTIR Spectra; Samples 1 and 3.

Figure 8 shows the $\alpha / \beta$ ratio progression during the fast reaction period for a range of temperatures. For each temperature, the observed $\alpha / \beta$ ratio remains constant throughout the reaction, indicating that both phases grow at the same rate after identical induction periods; the $\alpha / \beta$ ratio is temperature dependent. Identical induction times and growth rates are unlikely to result from two fundamentally different mechanisms over a range of temperatures, although it might be postulated that an impurity effect could result in identical induction periods if it controlled the formation of both types of nuclei. Although not proven, it was concluded that both $\alpha-$ and $\beta-\mathrm{Si}_{3} \mathrm{~N}_{4}$ phases probably form by vapor phase reactions between $\mathrm{Si}_{v}$ and $\mathrm{N}_{2}$, and that higher temperatures make nucleation of the $\beta$-phase more probable.

Nucleation models generally consider the surface to be invariant, which is not the case for this material since an amorphous silicon nitride layer grows continuously during the induction period. The thickness of the amorphous layer that forms in a $\mathrm{N}_{2}$ atmosphere at the end of the induction period, $\mathrm{l}^{*}$, is shown in Figure 9 for a range of nitriding temperatures. Two sets of experiments tested the effect of $I^{*}$ on the nucleation process. Exposure to forming gas $\left(95 \% \mathrm{~N}_{2}-\right.$ $5 \% \mathrm{H}_{2}$ ) decreased I" significantly, but did not affect the induction times. In another set, samples were held at temperatures that caused growth of the amorphous layer but not nucleation, and were then rapidly heated to a higher temperature where nucleation occurred. Samples exposed to two temperatures had both increased $I^{*}$ values and induction times in contrast to expectations based on models that relate the nucleation barrier to film thickness. These observations, in combination with the $\mathrm{I}^{*}$ temperature dependence, provide strong evidence that nucleation is not controlled by the growth of the amorphous silicon nitride layer. 


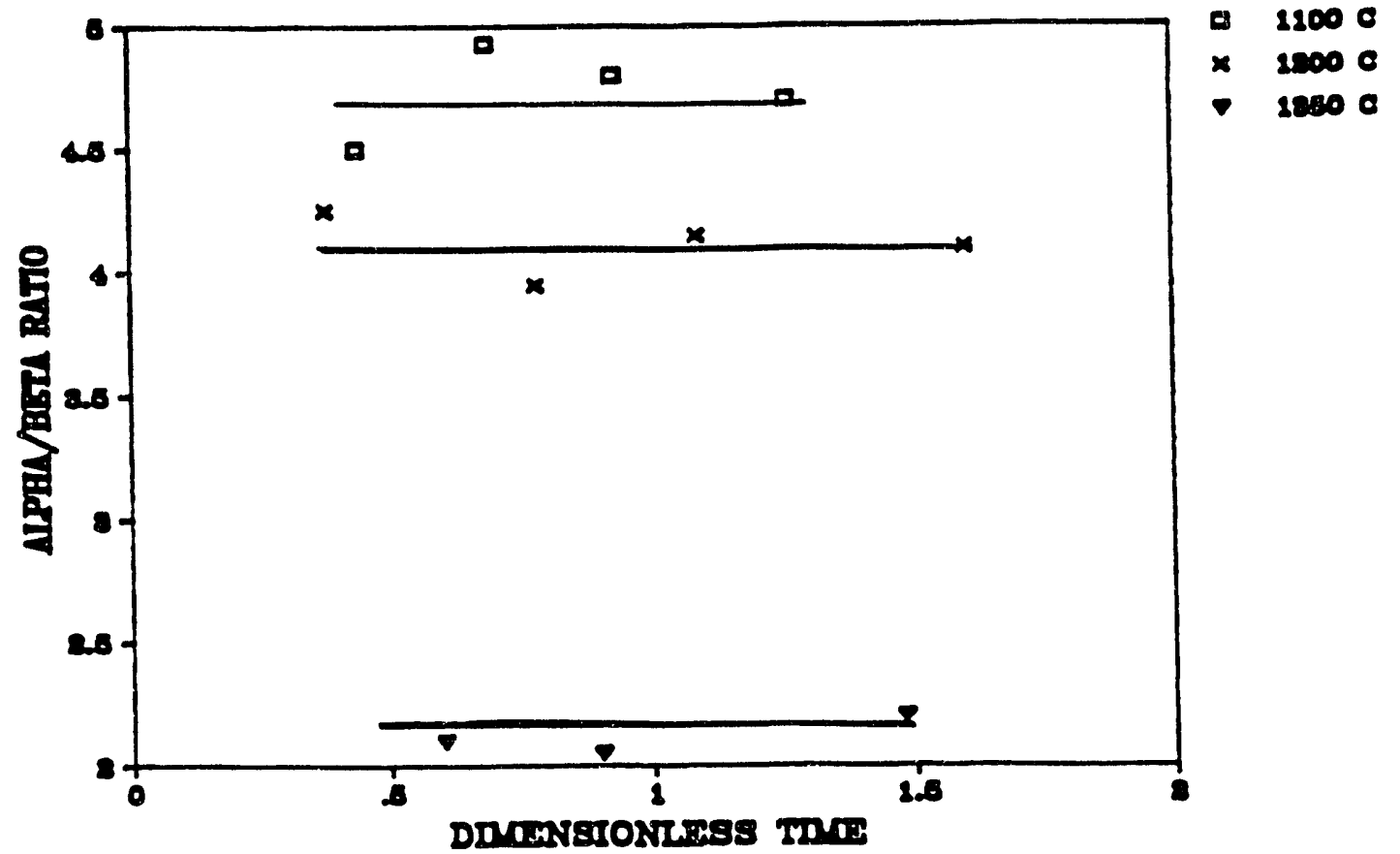

Figure 8. $\alpha / \beta$ ratio during the "fast" reaction, at different temperatures. The dimensionless time was obtained by dividing the actual time by the time at the end of the "fast" reaction (different at each temperature).

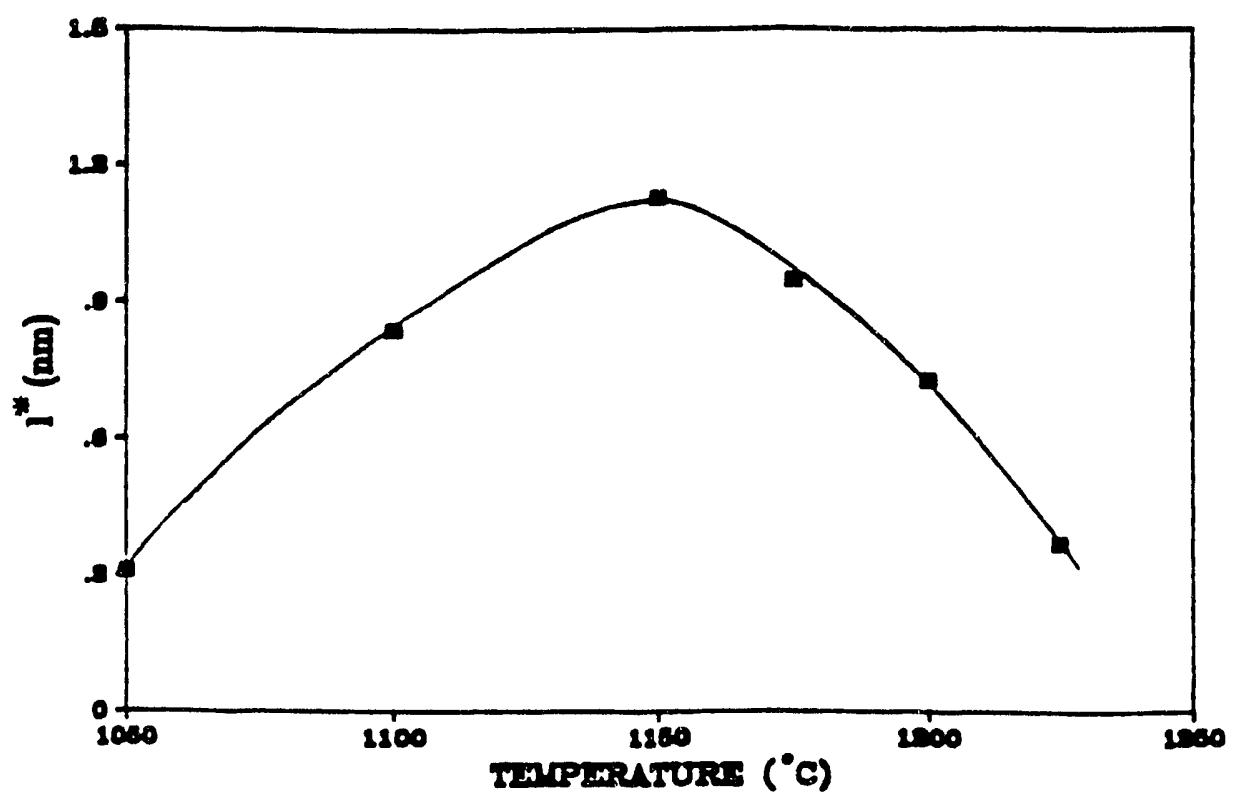

Figure 9. Plot of $l^{*}$ versus temperature $\left(l^{*}\right.$ is the thickness where nucleation is initiated).

TEM characterization of nitrided powders exhibited three general morphologies. These were:

1. Spherical particles which are similar to the starting Si powder, except that some neck growth occurred between touching particles. The samples which were interrupted prior to the fast reaction period $(1,2,3$ and 8) predominantly had this morphology. 
2. Spherical particles with several small angular crystallites attached to their surfaces. Samples fired just into the beginning of the fast reaction period $(4,5$ and 9$)$ exhibited coarsened Si particles both with and without the crystallites.

3. Agglomerated material in which resolution of detail was virtually impossible. Samples fired well into the fast reaction period $(6,7,10$ and

11) were mostly agglomerated.

Selected area diffraction analyses showed that the spherical particles were unreacted $\mathrm{Si}$ and that the angular crystallites were $\mathrm{Si}_{3} \mathrm{~N}_{4}$. A typical TEM photomicrograph of a sample taken just after the beginning of the fast reaction period is shown in Figure 10.

The morphology of the $\mathrm{Si}_{3} \mathrm{~N}_{4}$ crystallites shows that the $\mathrm{Si}_{3} \mathrm{~N}_{4}$ grows out and away from, or along, the Si particles rather than growing into them. This observation suggests that $\mathrm{Si}$ transport to a $\mathrm{Si}_{3} \mathrm{~N}_{4} /$ vapor growth interface, rather than nitrogen transport to a $\mathrm{Si} / \mathrm{Si}_{3} \mathrm{~N}_{4}$ interface, dominates the growth phase of the conversion process.
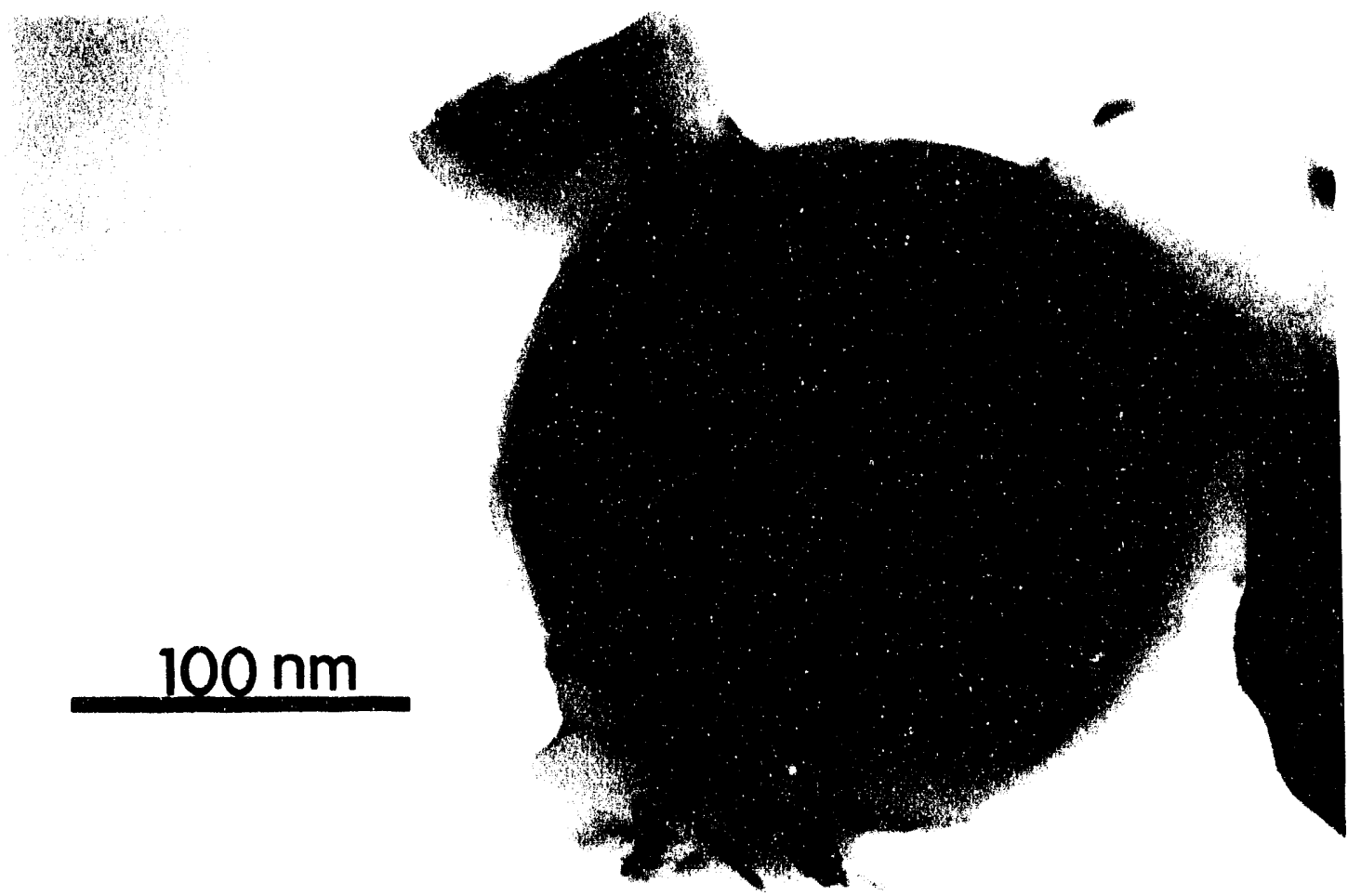

Figure 10. TEM micrograph, early "fast" reaction: Si sphere with several $\mathrm{Si}_{3} \mathrm{~N}_{4}$ crystals. 
TEM photomicrographs showed that the interface between the $\mathrm{Si}_{3} \mathrm{~N}_{4}$ and the $\mathrm{Si}$ phases was generally flat. This observation suggests that nucleation occurred preferentially with specific heteroepitaxial relationships and also that the development of these preferred nucleation planes may have taken place during the induction period. Extensive TEM diffraction analyses indicated that $\mathrm{Si}_{3} \mathrm{~N}_{4}$ growth generally occurred preferentially along [0001] directions, and that in most cases the (1120) $\mathrm{Si}_{3} \mathrm{~N}_{4}$ planes in both the $\alpha$ and $\beta$ phases were aligned with Si (110) planes. Nucleation rates should be affected significantly by both of these observations.

The induction period apparently occurs because the nucleation of $\mathrm{Si}_{3} \mathrm{~N}_{4}$ is inhibited. Three plausible explanations for this behavior were considered:

1. The induction time corresponds to the time it takes to form a steady-state distribution of subcritical embryos. The measured induction times fit an approximate expression for this relaxation time; however, the data is somewhat scattered, and some of the simplifying assumptions associated with the model are clearly incorrect.

2. The growth of a thin amorphous region around the silicon particles (several atom layers thick) reduces the surface free energy barrier to nucleation as the induction period proceeds. Experiments show that the thickness of this layer does not strictly control nucleation, and that the presence of hydrogen in the nitriding gas alters the layer thickness without changing the induction time. These observations are contrary to the idea that nucleation is controlled by a decreasing surface free energy barrier as the thickness of an amorphous layer increases, although the results are not completely conclusive.

3. The flat $\mathrm{Si} / \mathrm{Si}_{3} \mathrm{~N}_{4}$ interfaces seen by TEM indicate that some surface rearrangement must take place. These interfaces are consistent with heteroepitaxial relationships between the silicon and silicon nitride. The induction period could correspond to the time it takes for this rearrangement to take place (i.e. faceting).

Based on the results, it was concluded that the induction period is controlled by the surface rearrangement, by the nonsteady-state phenomena associated with the formation of subcritical embryos, or by both of these mechanisms in combination. However, the experimental evidence for these mechanisms, particularly the first two, are subject to some uncertainty.

Moulson's hypothesis ${ }^{1}$ that the $\alpha$ and $\beta$ phases form by Si vapor and Si solid mechanisms respectively, is inconsistent with the progression of the $\alpha / \beta$ ratio that was measured here. In this system, nucleation is more likely to occur from silicon vapor than by a direct reaction with the solid. This conclusion is consistent with the conceptual considerations that were discussed; it is also based on the measured evaporation rates, and on the observation that the 
induction time activation energy is close to the sublimation enthalpy for silicon. However, the flat $\mathrm{Si} / \mathrm{Si}_{3} \mathrm{~N}_{4}$ interfaces show that the reaction also involves atoms at the solid surface. This suggests that both solid and vapor silicon atoms are involved, although the majority of the reactant silicon atoms are probably transported through the vapor phase. Further complexities in the nucleation mechanism involve the presence of atomic versus molecular nitrogen, the variations in the $\mathrm{Si}_{1} / \mathrm{Si}_{3} \mathrm{~N}_{4}$ surface free energy that can occur during nitridation, and difficulty in defining the point where a small subcritical embryo becomes distinct from the amorphous surface layer. This collection of issues demonstrates that nucleation is more complex than a simple single step mechanism.

The longer induction times and lower "fast" reaction conversion that occurs when the initial $\mathrm{Si}-\mathrm{H}$ surface undergoes submonolayer oxygen contamination are consistent with the three nucleation mechanisms that were considered; the induction period data for these samples reinforces the conclusions that were obtained with non-oxidized samples. The longer induction times that occur at lower temperatures and with oxygen contamination suggest that a lower nucleation rate is responsible for decreasing conversion to $\mathrm{Si}_{3} \mathrm{~N}_{4}$ during the "fast" reaction.

\section{E. Rapid Growth and Complete Conversion}

Nitriding results showed that complete nitridation could be achieved at very rapid rates once nucleation had occurred and if the fast reaction period were not terminated prematurely. Experiments were undertaken to:

1. Identify the factors responsible for the end of the fast reaction period,

2. Identify the factors responsible for the termination of the nucleation process,

3. Determine the rate controlling mechanisms during the slow reaction period.

The rapid growth period might terminate because either too many or too few nuclei formed. In the first case, the Si powder surfaces would become completely covered by $\mathrm{Si}_{3} \mathrm{~N}_{4}$ so that the process would transition from $\mathrm{Si}$ vaporization to solid-state diffusion control. In the second, a limited number of $\mathrm{Si}_{3} \mathrm{~N}_{4}$ grains would grow along preferred growth directions until they impinged on adjacent grains.

Weight loss experiments in vacuum indicated that free Si surfaces were present throughout the fast reaction period based on agreement between calculated and measured weight loss rates. Also, microstructural changes that resulted when partially nitrided samples were intentionally heated to temperatures in excess of the Si melting point confirmed the presence of free Si surfaces. Several experiments demonstrated that it was unlikely that the amorphous 
silicon nitride layer either evaporated or dissolved in these two types of experiments. TEM and $X$-ray line broadening showed that the grain size increased slightly, but continuously, during the fast reaction period. The grain size in the preferred growth direction agreed with probable impingement distances $(\sim 100 \mathrm{~nm})$ in compacts made of $200-300 \mathrm{~nm}$ Si powders. This set of experiments showed that fast growth proceeded from a limited number of nuclei on Si particles that retained free Si surfaces. Under these conditions, silicon vaporization controls the overall reaction rate until growth is terminated by impingement.

One set of experiments designed to isolate the effects of temperature on nucleation and growth rates also revealed a competing effect. For instance, samples that were heated momentarily to $1150^{\circ} \mathrm{C}$ and then nitrided at $1100^{\circ} \mathrm{C}$ reacted to completion while samples that were heated only to $1100^{\circ} \mathrm{C}$ or $1125^{\circ} \mathrm{C}$ did not react to completion. These results indicate that the presence of the additional nuclei which formed at $1150^{\circ} \mathrm{C}$ facilitated the reaction. Other cycles that involved heating, cooling and reheating to the maximum- or an intermediate-temperature level, suggest the simultaneous formation of a $\mathrm{Si}_{3} \mathrm{~N}_{4}$ layer directly on the Si surfaces at the lower temperatures; the effect of the layer was reversed by heating to $1150^{\circ} \mathrm{C}$ or higher, but it inhibited the reaction for lower temperatures. These results show that more nuclei form at higher temperatures; above critical temperature levels preexisting nuclei can grow at high rates, but are inhibited from growing by a surface film at temperatures below the critical level.

No conclusive reason was identified for the termination of the nucleation process; however, geometric constraints must play a role. With the footprint that forms normal to the preferred growth direction, there is only room for between 5-50 nuclei per Si particle even without restrictions imposed by preferred heteroepitaxial planes. In most cases, we conclude that about $10 \mathrm{Si}_{3} \mathrm{~N}_{4}$ nuclei form per Si particle. Once they form at the beginning of the fast reaction period, apparently no further nuclei form even though fast growth is inhibited by impingement. Experiments with smaller Si particles achieved complete conversion during fast reaction under conditions that caused only partial conversion with the normal powders. The higher surface area per unit volume apparently allowed adequate numbers of nuclei to form.

Melting and evaporation experiments with samples held in the slow reaction period indicate that they no longer exhibit the free Si surfaces that existed throughout the fast reaction period. This coverage can result from either the formation of a silicon nitride film between the existing $\mathrm{Si}_{3} \mathrm{~N}_{4}$ grains or the growth of these grains in non-preferred directions. X-ray line broadening showed that some growth occurred during the slow reaction period. Reaction rates during the slow period might be justified by parabolic kinetics modified for the $22 \%$ volumetric expansion if diffusion is enhanced adequately by grain boundary pathways. The experiments show that the Si surfaces are covered by 
crystalline $\mathrm{Si}_{3} \mathrm{~N}_{4}$ during the slow reaction period, but they do not confirm the growth mechanism.

\section{F. The Effects of Low-Level Oxygen Contamination}

As already noted, the "reverse" composition gradients that were observed in the TGA nitrided samples are caused by oxygen contamination from the $\mathrm{N}_{2}$. This effect was further studied by comparing results with standard ultrahigh-purity $\mathrm{N}_{2}$ (< $10 \mathrm{ppm}$ total impurities) to results where the gas was first passed through a gas purifier which brought the total $\mathrm{O}_{2}$ and $\mathrm{H}_{2} \mathrm{O}$ impurities down to below 10 $\mathrm{ppb}$. These will be referred to as the unpurified and purified gases, respectively. Since the process is very sensitive to the purity of the environment, the entire TGA furnace and the associated plumbing was leak checked with a He leak detector down to $10^{-8} \mathrm{mbar} / \mathrm{s} . \mathrm{A} \mathrm{ZrO}_{2}$ based $\mathrm{O}_{2}$ analyzer was used to monitor the $\mathrm{O}_{2}$ content of the exhaust gases from the furnace. This instrument was modified because the oxygen levels in the purified gas and the exhaust gas were below the manufacturer's specifications.

The results with the two gas purities are compared in Tables 3 and 4 . Similar to previous findings, 40,41 the results of this study illustrate the effects that small amounts of oxygen have on the nitriding kinetics of high purity Si powders. To varying degrees, increased oxygen content and/or longer exposures due to slower heating rates generally cause longer induction times, lower reaction rates, lower overall conversion at the end of the "fast" reaction, and increased $\alpha / \beta$ ratios. These effects are more pronounced at lower temperatures (i.e., $1100-1200^{\circ} \mathrm{C}$ ), and are particularly evident at the exterior surfaces of the porous samples.

The external surface of samples formed at $1100^{\circ} \mathrm{C}$ w.th unpurified gas are the only conditions where crystalline $\mathrm{Si}_{3} \mathrm{~N}_{4}$ did not form. This indicates that a threshold was crossed, where $\mathrm{Si}_{3} \mathrm{~N}_{4}$ was observed in the interior regions under these conditions (i.e., lower $\mathrm{PO}_{2}$ ), at the external surfaces with purifed gas (i.e., lower $\mathrm{PO}_{2}$ ), and at the external surfaces of all of the samples formed at higher temperatures. Previous results showed that the nucleation of $\mathrm{Si}_{3} \mathrm{~N}_{4}$ is completely inhibited until the beginning of the fast reaction period. 14,15 Based on the longer induction times and the corresponding lower conversion to $\mathrm{Si}_{3} \mathrm{~N}_{4}$ that were observed here and previously, 40 oxygen contamination inhibits the nucleation of crystalline $\mathrm{Si}_{3} \mathrm{~N}_{4}$. Thus the absence of $\mathrm{Si}_{3} \mathrm{~N}_{4}$ at $1100^{\circ} \mathrm{C}$ with the unpurified gas is probably related to kinetic factors that limit nucleation. The observation that $\mathrm{Si}_{3} \mathrm{~N}_{4}$ forms in the middle of these samples indicates that this kinetic limitation is related to the oxidation that occurs near the outer surfaces.

In general, active and passive oxidation refer to the formation of gas and solid phase oxide products, respectively. The thermodynamics of Si oxidation were originally described by Wagner. ${ }^{42}$ Several more recent papers have considered active and passive oxidation in the Si-O-N system in detail. 43-45 A similar analysis was performed for the experimental conditions that were studied, and 
Table 3. Summary of Nitriding Kinetics Results

$\% \quad$ Max.

$\begin{array}{cclccc}\begin{array}{c}\text { Temp } \\ { }^{\circ} \mathrm{C}\end{array} & \begin{array}{c}\text { Htg Rate } \\ { }^{\circ} \mathrm{C} / \mathrm{min}\end{array} & \text { Gas Purity } & \begin{array}{c}\text { Conversion } \\ \text { overall }\end{array} & \begin{array}{c}\text { Induction } \\ \text { Period (min) }\end{array} & \begin{array}{c}\text { Rkn. Rate } \\ \% / \mathrm{min}\end{array} \\ 1100 & 100 & \text { Unpurified } & 82 & 16 & 4.5 \\ 1100 & 100 & \text { Purified } & 88 & 16 & 5 \\ 1150 & 100 & \text { Unpurified } & 89 & 2 & 18 \\ 1150 & 100 & \text { Purified } & 93 & 1 & 20 \\ 1200 & 100 & \text { Unpurified } & 93 & 0 & 23 \\ 1200 & 100 & \text { Purified } & 92 & 0 & 27 \\ 1110 & 5 & \text { Unpurified } & 82 & 40 & 2 \\ 1110 & 5 & \text { Purified } & 88 & 28 & 2.5\end{array}$

Table 4. Summary of X-ray Diffraction Results

$\begin{array}{cclcccc}\begin{array}{c}\text { Temp } \\ { }^{\circ} \mathrm{C}\end{array} & \begin{array}{c}\text { Htg rate } \\ { }^{\circ} \mathrm{C} / \mathrm{min}\end{array} & \text { Gas Purity } & \begin{array}{c}\% \\ \text { Conversion } \\ \text { surface }\end{array} & \begin{array}{c}\% \\ \text { Conversion } \\ \text { center }\end{array} & \begin{array}{c}\alpha / \beta \\ \text { surface }\end{array} & \begin{array}{c}\alpha / \beta \\ \text { center }\end{array} \\ 1100 & 100 & \text { Unpurified } & 0 & 91 & -- & 6 \\ 1100 & 100 & \text { Purified } & 53 & 93 & 7 & 3 \\ 1150 & 100 & \text { Unpurified } & 66 & 90 & 6 & 5 \\ 1150 & 100 & \text { Purified } & 69 & 94 & 7 & 4 \\ 1200 & 100 & \text { Unpurified } & 79 & 98 & 4 & 3 \\ 1200 & 100 & \text { Purified } & 74 & 97 & 3 & 3 \\ 1110 & 5 & \text { Unpurified } & 20 & 92 & -- & 17 \\ 1110 & 5 & \text { Purified } & 74 & 87 & 16 & 9\end{array}$

it was determined that passive oxidation of silicon is thermodynamically favorable for the one set of conditions where $\mathrm{Si}_{3} \mathrm{~N}_{4}$ does not form $\left(1100^{\circ} \mathrm{C}\right.$, unpurified gas, external surfaces), and that active oxidation of silicon is thermodynamically favorable for the other experimental conditions. ${ }^{46}$

The hypothesis that $\mathrm{Si}_{3} \mathrm{~N}_{4}$ nucleation can not occur when passive oxidation of $\mathrm{Si}$ is favorable is a reasonable explanation for the experimental observations. In general, $\mathrm{O}_{2}$ is much more reactive than $\mathrm{N}_{2}$. Previous work with these silane derived powders at lower temperatures shows that nitrogen adsorbs many orders of magnitude slower than oxygen. 43 Thus, compared to oxygen, nitrogen is almost inert during the early part of the process, such that passive oxidation of $\mathrm{Si}$ is important, instead of the passive oxidation of a nitride phase $\left(\mathrm{Si}_{3} \mathrm{~N}_{4}\right.$ or $\left.\mathrm{Si}_{2} \mathrm{~N}_{2} \mathrm{O}\right)$. 
Moulson proposed that nitridation can only occur if active oxidation removes the native oxide layer that is present on conventional Si powders. ${ }^{1}$ With these silane derived powders the situation is considerably different because a native oxide layer does not exist; however, active oxidation is apparently still necessary. It is possible that the adsorbed oxygen must be removed for nucleation to occur, similar to the removal of thicker oxide layers on conventional powders. This is one possible explanation for the induction period, however, with only submonolayer contamination of the Si surfaces, this process is mechanistically different from Moulson's description of the removal of a thicker oxide layer. During the induction period, the observed TGA weight gain shows that sorne nitridation is occurring; however, the formation of crystalline material does not occur until the onset of the "fast" reaction.14,15 It is possible that surface oxygen inhibits nucleation, and that oxygen desorption occurs during the induction period at a slower rate than nitridation, such that a weight gain is observed. This suggests that the formation of crystalline $\mathrm{Si}_{3} \mathrm{~N}_{4}$ is a complex process, where nitrogen adsorption, oxygen desorption, and nucleation are all important kinetic processes.

If $\mathrm{Si}_{3} \mathrm{~N}_{4}$ nucleation requires active oxidation, then with a given oxygen contamination level, the temperature must exceed the passive to active transition temperature. It might be beneficial to conduct nucleation at a higher temperature for a short period of time, followed by continued nitridation at a lower temperature. This approach is particularly attractive for composite applications where prolonged exposures at high temperatures are problematic. Preliminary investigations into this approach using silane derived powders have demonstrated this possibility. ${ }^{37}$ In these experiments the nitridation was conducted at $1150^{\circ} \mathrm{C}$ until TGA measurements showed that the "fast" reaction had begun, at which point the temperature was lowered to 1100 or $1050^{\circ} \mathrm{C}$. Compared to isothermal nitridation at 1100 or $1050^{\circ} \mathrm{C}$, this two-step approach produced significantly higher conversion to $\mathrm{Si}_{3} \mathrm{~N}_{4}$.

\section{G. The Effects of Binder and Solvent Exposures}

Research described in the preceeding sections indicates that submonolayer oxygen contamination of the powder surfaces inhibits the nucleation of $\mathrm{Si}_{3} \mathrm{~N}_{4}$. To produce material with practical sizes, shapes, and densities, binders and solvents must be used. Similar to oxidation, these additives affect the surface chemistry and nitridation kinetics. 47 Optimum binders and solvents will minimize adverse changes in the powders' surface chemistry. These changes can result directly through reactions with both the binder and solvent, as well as the ambient atmosphere used for binder burnout. Indirectly, the heating schedules used for binder burnout and other processing requirements attributable to the binder can make the powders vulnerable to oxygen and other agents. Both types of adverse effects must be minimized.

The effects of exposures to candidate solvents and binders on the nitriding kinetics of the $\mathrm{SiH}_{4}$-derived Si powders have been investigated. Table 5 
summarizes the polymer and solvent systems that were investigated. Polyethylene (PE) and polystyrene (PS) are both simple hydrocarbons which burn out cleanly and are used in injection molding. $\mathrm{Qpac}^{\mathrm{TM}}$ is a binder that has been designed to burn out cleanly in a nitrogen atmosphere. 48 The tetrafluoroethylene/polystyrene (TFE/PS) system has been used in the fabrication of SiC fiber-reinforced RBSN. 49 The polysilazane (PSZ) was interesting for several reasons. PSZ forms a Si-, $\mathrm{N}$ - and $\mathrm{C}$-containing ceramic product upon pyrolysis 50 that is space-filling and evolves smaller amounts of gaseous products which need to escape during the burnout. These features should enhance the microstructural quality. The PSZ residue may protect the silicon particle surfaces and also may offer an alternative reaction site for parallel nucleation of crystalline $\mathrm{Si}_{3} \mathrm{~N}_{4}$. One concern is that PSZ films may block the silicon vapor transport and therefore limit the fast reaction rate. 37

Table 5. Polymer/Solvent Systems Investigated

\begin{tabular}{|c|c|c|c|c|}
\hline Binder & Eormula & Solvent & $\begin{array}{c}\text { Stab Wt } \\
I\left({ }^{\circ} \mathrm{C}\right)\end{array}$ & $\begin{array}{c}\operatorname{Max} T \\
\left.{ }^{\circ} \mathrm{C}\right) \\
\end{array}$ \\
\hline Polyethylene(PE)* & {$\left[\mathrm{CH}_{2}\right]_{n}$} & - & 490 & 600 \\
\hline QPAC ${ }^{\mathrm{TM}} 40^{* *}$ & {$\left[\mathrm{C}_{4} \mathrm{H}_{6} \mathrm{O}_{3}\right]_{\mathrm{n}}$} & Methanol & 500 & 500 \\
\hline Polystyrene(PS) ${ }^{\star \star \star}$ & {$\left[\mathrm{C}_{6} \mathrm{H}_{5} \mathrm{CHCH}_{2}\right]_{n}$} & Xylene & 460 & 600 \\
\hline $\begin{array}{l}\text { Teflon(TFE) } \\
\text { Polystyrene } e^{\star \star * \star *}+\end{array}$ & {$\left[\mathrm{CF}_{2}\right]_{\mathrm{n}}$} & Stoddard & 580 & 600 \\
\hline $\begin{array}{l}\text { Polysilazane } \\
\text { (PSZ)*****}\end{array}$ & $\left|\begin{array}{l}{\left[\mathrm{CH}_{3} \mathrm{SiHNH}\right]_{0.39}} \\
\left.\left[\mathrm{CH}_{3} \mathrm{SiHNCH}\right]_{3}\right]_{0.04} \\
{\left[\mathrm{CH}_{3} \mathrm{SiN}\right]_{0.57}}\end{array}\right|$ & Toluene & $>75^{n}$ & 750 \\
\hline
\end{tabular}

* A735 Polyethylene wax, Allied Signal Co., Morristown, NJ 07960

** QPACTM 40, Lot 10021-30, Air Products and Chemicals, Inc., Emmaus, PA 18049

*** Polystyrene - supplied by NASA-Lewis

****Teflon@, E.I. DuPont de Nemours \& Co., Inc., Wilmington, DE 19898

***** Polysilazane M. Wt. 6000-8000, proprietary composition, Ethyl Corp., Baton Rouge, LA 70801; indicated composition proposed by Wiseman for a similar polymer 
Several general observations can be made about the effect of the investigated polymers on the nitriding kinetics of the high purity Si powders. ${ }^{47}$ These comments apply to the following groups of polymers.

PE/PS: These relatively simple hydrocarbons burn out cleaner, and only delay the onset of the nitriding reaction. For $P E$, it appears that this results from some reduction in the nucleation rate (based on the longer induction time, the slightly lower conversion, and the estimated number of nuclei per particle). PS, however, demonstrated similar nitriding results apparently with a relatively high number of nuclei. These results suggest that surface contamination with simple hydrocarbons does not inhibit nitridation as severely as surface contamination from oxygen, alcohol, and the other polymers that were studied.

TFE/Qpac: These binders contain oxygen and fluorine in addition to carbon and hydrogen. These apparently cause more serious surface contamination which leads to incomplete conversion at low temperatures. The data suggest excessive nuclei are responsible for the inhibited reactions despite an initial nucleation barrier. A significant amount of $\mathrm{Si}$ is lost by vaporization from the TFE samples.

PSZ: $\quad$ By design, the Si-N-C-H polymer leaves $75 \%$ residue behind after binder burnout, which significantly alters the particles' surfaces. The relatively high number of estimated nuclei per particle suggests that $\mathrm{Si}_{3} \mathrm{~N}_{4}$ might actually nucleate within this residue.

At the end of binder burnout there is little $\mathrm{Si}-\mathrm{N}$ bonding and the protective $\mathrm{Si}-\mathrm{H}$ surface is lost, leaving powders vulnerable to oxidation. 37,47 Slow heating rates and lengthened induction periods resulted in significant oxidation. Use of gettered nitrogen, correct polymers and faster heating rates, when the binder will allow, will minimize this oxidation. Other residues from the polymers can accentuate or negate the effect of oxygen on nitriding kinetics. Oxidation does not effectively retard nitriding kinetics when there is hydrocarbon bonding on the silicon surface. The same effect has been observed previously when free carbon is present in the form of high purity carbon-rich SiC. 47 In contrast, residues from more complex polymers significantly retard nitriding kinetics, apparently by accentuating oxygen's effects.

Several additional phenomenological generalizations can be made. If samples with uncertain initial binder content are excluded, the weight gained during the induction period is proportional to the length of the induction period. The reaction kinetics suggest several optimizations which may be necessary to achieve rapid conversion at minimum temperatures. The number of nuclei which form per silicon particle should be $<100$. An induction period weight gain of about one weight $\%$ maximizes the fast reaction rate. A peak reaction 
rate of at least $15 \%$ per minute seems necessary to achieve full conversion of the $\sim 0.25 \mu \mathrm{m}$ diameter Si particles during the fast period at $1250^{\circ} \mathrm{C}$. Polymer exposed samples showing incomplete conversion at $1250^{\circ} \mathrm{C}$ nitride to completion after the $1400^{\circ} \mathrm{C} 10 \mathrm{~h}$ schedules. It has been shown that these extended schedules do not damage the SiC reinforcements. 51 In general, these results indicate that reasonable nitridation rates at relatively low temperatures is possible with the use of the proper polymers.

\section{H. Microstructural Characterizations}

Detailed microstructural characterizations of fully nitrided samples were made to identify distinguishing characteristics between high- and low-K $K_{1 C}$ samples. 23,52 RBSN samples as well as constituent powders were examined by SEMFEG, TEM, BET, XRD, and $\mathrm{Hg}$-porosimetry to define microstructural and fractographic characteristics. Though focused on interpreting differences in observed mechanical properties, the results apply equally to the nitriding process which defined the final microstructures. The results are summarized in Table 6.

Table 6. Summary of characteristics for high and low fracture toughness RBSN. 23,52

Prenitrided (Si) Bulk Density (\%)

Nitrided Bulk Density (\%)

Extent Nitrided by XRD (\%)

Extent Nitrided by wt. gain (\%)

$\alpha / \beta$ by XRD

Surface Area by Microscopy $\left(\mathrm{m}^{2} / \mathrm{g}\right)$

Surface Area by BET $\left(\mathrm{m}^{2} / \mathrm{g}\right)$

Eq. Spherical Diam. by BET $(\mu \mathrm{m})$

Grain Size by XRD $(\mathrm{nm})$

Lattice Distortion by XRD (\%)

Grain Size by TEM $(\mathrm{nm})$

\begin{tabular}{cc} 
High K K & Low $K_{I C}$ \\
\hline 62 & 59 \\
76 & 75 \\
$>98$ & $>98$ \\
96.3 & 96.8 \\
4.1 & 3.0 \\
5.6 & 6.6 \\
2.9 & 3.3 \\
0.66 & 0.56
\end{tabular}

$65.7 \pm 4.3$

$45.6 \pm 8.1$

$0.20 \pm 0.02$

114
$0.15 \pm 0.03$

76

Most of these results are within ranges reported previously for RBSN made from $\mathrm{SiH}_{4}$-derived $\mathrm{Si}$ powders. The specific surface areas confirm that the interiors of the samples are generally open to the exterior surfaces and that the RSBN microstructure retains the small characteristic dimensions of the starting powders. TEM micrographs show that the RBSN is characterized by a highly tortuous structure with interconnected, cocontinuous pore and solid phases. In most micrographs, it is almost possible to traverse the figure by following a path through either the pore or solid phase. Pore shapes and sizes vary. With some license, the porosity can be grouped into somewhat irregular larger pores and narrower pore channels. The largest dimension of most pores falls in the range of $20 \mathrm{~nm}$ to $250 \mathrm{~nm}$, with an average of approximately $130 \mathrm{~nm}$. Pore channels 
vary in width within an approximate range of $10 \mathrm{~nm}$ to $40 \mathrm{~nm}$. The solid phase appears as an interconnected, high aspect-ratio ribbon-like structure that presumably extends in all directions throughout the microstructure. The widths of the solid phase are in the range of $80-400 \mathrm{~nm}$. From these micrographs, it is not possible to conclude whether the solid phase has a cylindrical, strut-like structure, or whether it is more like a cell wall and extends in two dimensions.

The mean spacing between larger pores was determined from highlighted micrographs by manual analysis of line intercepts. ${ }^{53}$ These essentially identical interpore dimensions are 0.54 and $0.55 \mu \mathrm{m}$ for the high and low toughness RBSN materials respectively. Using the actual Si mean particle diameters and bulk Si density values, the Si interparticle spacings in the prenitrided pellets for the high and low toughness materials are 0.41 and 0.39 $\mu \mathrm{m}$ respectively. 54 The agreement between interpore spacings and the estimated values for mean center-to-center spacings of Si particles in the green parts suggests that the larger pores are relics of the Si particles, and that the reaction product (containing interconnected channels) initially formed within the interstitial regions between the original Si particles. The difference between the mean pore diameter defined from micrographs $(130 \mathrm{~nm})$ and the mean $\mathrm{Si}$ particle size $(300 \mathrm{~nm})$ results from the necessary formation of RBSN within the volumes occupied by the Si particles since the volume of the interstitial space is inadequate to accommodate all of the reaction product. Assuming that the number of pores within the RBSN equals the number of Si particles in the unnitrided sample, these diameter ratios indicate that approximately half of the total RBSN porosity is located within the interstitial space between the original Si particles.

A mixture of material taken from high and low toughness fragments was characterized by $\mathrm{Hg}$-porosimetry. The bulk density of the combined sample was $2.44 \mathrm{~g} / \mathrm{cc}$, or $76.7 \%$ of theoretical corresponding to $23.3 \%$ porosity. This density value essentially equals the values cited in Table 6 that were defined from physical dimensions of polished samples. The revealed porosity was observed to occur in two pore size ranges that were partitioned as follows:

$\begin{array}{lc}\text { Pore Diameter }(\mathrm{um}) & \text { \% of Bulk Volume } \\ 6.0-6.4 & 0.05 \\ 0.01-0.04 & 10.6\end{array}$

The large pore channels evident in the low toughness RBSN were not revealed by $\mathrm{Hg}$-porosimetry, showing that they are not interconnected with the exterior surfaces through large diameter paths. Also, $12.65 \%$ of the bulk volume, over half the total porosity, was not revealed by $\mathrm{Hg}$-porosimetry, indicating that the residual porosity is either accessed through pore channels smaller than 0.01 $\mu \mathrm{m}$, or it is closed. If interconnected pores are assumed, neither the volume fractions nor the sizes of the pores revealed by $\mathrm{Hg}$-porosimetry are consistent 
with TEM and SEM microscopy observations, unless it is concluded that the pathways to half of the larger pores are defined by extremely narrow $(<10 \mathrm{~nm})$ necks in the pore channels. He-pycnometry measurements of as-nitrided and surface ground samples both indicate skeletal densities are $\sim 85 \%$, indicating that approximately $15 \%$ of the RBSN sample volumes, $>50 \%$ of the total pore volumes, are made up of closed pores. Similar discrepancies in densities have been observed with high surface-area sol-gel materials which were attributed to the effects of adsorbed species. 55 The differences between specific surface areas defined by BET and microstructural analysis given in Table 6 could be caused by the existence of pores in the RBSN that closed after cooling and exposure to the ambient. Because it is extremely unlikely that significant fractions of these samples could be isolated from the exterior surfaces under nitriding conditions based on the observed nitriding kinetics, if pore closure occurred, it could have happened only after nitridation. TEM micrographs revealed an $\sim 10 \mathrm{~nm}$ thick amorphous layer on the surfaces of the pores and channels which appeared to block occasional channels. In one case, a $135 \mathrm{x}$ $180 \mathrm{~nm}$ grain of residual Si was identified. As was expected from proposed models of the nitriding process, 37,40 the Si grain was surrounded by numerous $\mathrm{Si}_{3} \mathrm{~N}_{4}$ grains and three pores which create the exposed Si surfaces needed for rapid, low temperature nitridation. The fact that no channels were connected to these pores may be responsible for its failure to convert to $\mathrm{Si}_{3} \mathrm{~N}_{4}$. The details of the pore structure will have important consequences for properties and nitriding kinetics.

Grain sizes have been defined by $X$-ray line broadening and from TEM micrographs. The TEM results summarized in Table 6 represent average intercept lengths on the micrographs. Because TEM foil thicknesses are roughly equal to the observed grain sizes, the relationship between intercept length and grain size does not match either of two limiting cases. If the foil was much thinner than the grain size, it would approximate a metallographic section, and the mean grain diameter would equal $3 / 2$ the mean intercept length. 56 If the foil was much thicker than the grain size, the observed intercepts would equal the grain diameters. Thus, true grain sizes lie between 1.0 and 1.5 times the values cited in Table 6 .

The TEM derived intercept lengths have the same relative values for high and low toughness RBSN sample types as the grain sizes observed by $X$-ray line broadening in Table 6, but are almost two times larger. Using the factor of 1.5 would increase this discrepancy to a factor of 3 . The difference between TEM and $X$-ray derived grain sizes probably results largely from faults in the grains, since the $X$-ray technique will yield the diameters of fault-free coherent scattering domains, and TEM intercepts measured the diameters of grains which included faults. 


\section{CONClusions}

Although several important unanswered questions remain, a relatively complete phenomenological description of the nitridation process with high-purity $\mathrm{Si}$ powders has been developed. An understanding of the mechanisms which permit rapid, low temperature nitriding kinetics with as-synthesized powders provided the basis for achieving similar nitriding results with practical atmospheric exposures, polymeric binders and solvents. This research provides the basis for realizing the full potential of reaction formed ceramic materials.

The rapid, low temperature nitriding result from subtle surface chemistry effects on the Si particles beginning with the loss of chemisorbed hydrogen and the sequential formation of very thin amorphous silic on nitride layers. Alpha and beta $\mathrm{Si}_{3} \mathrm{~N}_{4}$ nuclei form only over a very short period of time as the amorphous $\mathrm{Si}-\mathrm{N}$ layer disappears, evidently under local conditions which favor active oxidation. The number of nuclei which form, and their formation rate, are extremely sensitive to trace levels of oxygen introduced either with the nitriding gas or from exposures to water contaminated solvents. This effect can result in an unusual reverse reaction gradient in partially reacted parts that is caused by the interior regions being more reactive because oxygen contaminates are stripped from the gas by the outer regions. Carbonaceous residues can largely offset the kinetic effects of oxygen contamination.

A principal conclusion of this research is that rapid complete conversion to $\mathrm{Si}_{3} \mathrm{~N}_{4}$ during the fast reaction can be inhibited when either too few, or too many nuclei form on the Si particies. When too many form, free evaporation of Si from the surfaces of the Si particles is precluded. Alternatively, the rapid reaction period terminates prematurely when an insufficient number of nuclei form. Under the latter conditions, it is likely that the fast reaction period ends when $\mathrm{Si}_{3} \mathrm{~N}_{4}$ grains growing along preferred directions impinge on adjacent grains. Nucleation was shown to occur only with specific heteroepitaxial relationships between the two phases, and only after flats developed on the Si particles. The initial nucleation process probably stops because the existing $\mathrm{Si}_{3} \mathrm{~N}_{4}$ crystals create geometrical limitations on additional nucleation. Optimally, approximately $10 \mathrm{Si}_{3} \mathrm{~N}_{4}$ nuclei form per Si particle under conditions permitting rapid and complete nitridation. Nitridation during the slow reaction period appears to progress by both continued reaction of the non-preferred $\mathrm{Si}_{3} \mathrm{~N}_{4}$ growth interfaces and direct nitridation of the remaining Si/vapor interfaces. 


\section{REFERENCES}

1. A.J. Moulson, Reaction Bonded Silicon Nitride: its Formation and Properties, J. MATER. SCI. 14, 1017-51 (1979).

2. H.M. Jennings, B.J. Dalgleish and P.L. Pratt, Reactions Between Silicon and Nitrogen Part 2 Microstructure, J. MATER. SCI. 23, 2573-83 (1988).

3. F.D. Gac, Synthesis and Characterization of VLS Silicon Carbide Whisker Reinforced Reaction Bended Silicen Nitride, Ph.D. Thesis, University of Washington, 1989.

4. J.A. Mangels, Effect of $\mathrm{H}_{2}-\mathrm{N}_{2}$ Nitriding Atmospheres on the Properties of Reaction-Sintered $\mathrm{Si}_{3} \mathrm{~N}_{4}$, J. AM. CERAM. SOC. 58 [7-8] 354-355 (1975).

5. J.A. Mangels, Effect of Rate-Controlled Nitriding and Nitriding Atmospheres on the Formation of Reaction-Bonded $\mathrm{Si}_{3} \mathrm{~N}_{4}, \mathrm{AM}$. CERAM. SOC. BULL. 60 [6] 613-617 (1981).

6. D.R. Messier, P. Wong, and A.E. Ingram, Effect of Oxygen Impurities on the Nitridation of High-Purity Silicon, J. AM. CERAM. SOC. 56 [3] 171-172 (1973).

7. D.R. Messier, and P. Wong, Kinetics of Nitridation of Si Powder Compacts, J. AM. CERAM. SOC. 56 [9] 480-485 (1973).

8. D.R. Messier, and P. Wong, Ceramics for High Performance Applications, p. 181 (1974).

9. P. Wong, and D.R. Messier, Procedure for Fabrication of $\mathrm{Si}_{3} \mathrm{~N}_{4}$ by RateControlled Reaction Sintering, AM. CERAM. SOC. BULL. 57 [5] 525-526 (1978).

10. S.C. Danforth, and M.H. Richman, Strength and Fracture Toughness of Reaction-Bonded $\mathrm{Si}_{3} N_{4}$, AM. CERAM. SOC. BULL. 62 [4] 501-504 (1983).

11. W.R. Cannon, S.C. Danforth, J.H. Flint, J.S. Haggerty, and R.A. Marra, Sinterable Ceramic Powders from Laser Driven Reactions, Part I: Process Description and Modelling, J. AM. CERAM. SOC. 65 [7] 324-330 (1982).

12. W.R. Cannon, S.C. Danforth, J.H. Flint, J.S. Haggerty, and R.A. Marra, Sinterable Ceramic Powders from Laser Driven Reactions, Part II: Powder Characteristics and Process Variables, J. AM. CERAM. SOC. 65 [7] 330-335 (1982).

13. J.H. Flint, and J.S. Haggerty, Models for Synthesis of Ceramic Powders by Vapor Phase Reactions, First International Conference on Ceramic Powder Processing Science, Orlando, FL, November 1987, published in Ceramic Iransactions. Vol. 1. Ceramic Powder Science 1 . A, eds. Gary L. Messing, Edwin R. Fuller, Ir., and Hans Hausner, American Ceramic Society, Westerville, OH, pp. 244-252.

14. B.W. Sheldon, and J.S. Haggerty, The Nitridation of High Purity, LaserSynthesized Silicon Powder to Form Reaction Bonded Silicon Nitride, 12th Annual Conference on Composites and Advanced Ceramics, American Ceramic Society, Cocoa Beach, FL, January 1988, published in CERAM. ENG. SCI. PROC. 9 [7-8] 1061-72 (1988). 
15. B.W. Sheldon, and J.S. Haggerty, Formation of Reaction Bonded $\mathrm{Si}_{3} \mathrm{~N}_{4}$ at Low Temperatures and in Short Times, 13th Annual Conference on Composites and Advanced Ceramics, American Ceramic Society, Cocoa Beach, FL, January 1989, published in CERAM. ENG. SCI. Proc. 10 [7-8] 784-793 (1989).

16. J.S. Haggerty, G. Garvey, J-M. Lihrmann, and J.E. Ritter, Processing and Properties of Reaction Bonded Silicon Nitride Made from Laser Synthesized Silicon Powders, pp. 51-61 in Defect Properties and Processing of High-Technology Materials, Proceedings of the Materials Research Society, 1986. Edited by Y. Chen, W.D. Kingery, and R.J. Stokes. Materials Research Society, Pittsburgh, PA.

17. J.S. Haggerty, J. Flint, G. Garvey, J-M. Lihrmann, and J.E. Ritter, High Strength Oxidation Resistant Reaction Bonded Silicon Nitride from LaserSynthesized Silicon Powder, pp. 147-54 in Ceramic Materials and Components for Engines. Edited by W. Bunk and H. Hauser. Verlag Deutsche Keramische Gesellschaft, Saarbrüken, FRG, April 1986.

18. A. Lightfoot, F.M. Yee, C. Ker, B. Sheldon, and J.S. Haggerty, Processing of Reaction Bonded $\mathrm{Si}_{3} \mathrm{~N}_{4}-\mathrm{SiC}$ Composites, in 91st Annual Meeting, American Ceramic Society, Indianapolis, IN, April 1989.

19. Raytheon Company, Reaction Bonded Silicon Nitride for Endoatmospheric Hypersonic Radome Technology, 1992 Fourth Quarterly Report, U.S. Army Contract DASG 60-91-C-0124.

20. J.S. Haggerty, A. Lightfoot, J.E. Ritter, and S.V. Nair, High Strength, Porous, Brittle Materials, Materials Research Society 1990 Fall Meeting, Boston, MA, November 1990, published in MAT. RES. SOC. SYMP. PROC. Vol. 207, pp. 71-76 (1991).

21. P.A. Gennari, Eailure Analysis of Silane-Originating Reaction Bonded Silicon Nitride with and without a Pre-Oxidation Treatment, M.S. Thesis. University of Massachusetts, Amherst, MA, Sept. 1988.

22. C. Ker, MIT, unpublished results.

23. A. Lightfoot, L. Ewart, J. Haggerty, Z.Q. Cai, J. Ritter, and S. Nair, Processing and Properties of SiC Whisker- and Particulate-Reinforced Reaction Bonded $\mathrm{Si}_{3} \mathrm{~N}_{4}$, 15th Annual Conference on Composites and Advanced Ceramics, American Ceramic Society, Cocoa Beach, FL, January 1991, published in CERAM. ENG. SCI. PROC. 12 [7-8] 1265-1291 (1991).

24. J. Heinrich, Nitridation of Silicon under High Pressure, ADV. CERAM. MATLS. 2 [3A] 239-42 (1987).

25. R.T. Bhatt, Mechanical Properties of SiC Fiber-Reinforced ReactionBonded $\mathrm{Si}_{3} \mathrm{~N}_{4}$ Composites, NASA Technical Report 85-C-14, July 1985.

co. R.T. Bhatt, Effects of Fabrication Conditions on the Properties of SiC Fiber Reinforced Reaction-Bonded Silicon Nitride Matrix Composites (SiC / RBSN), NASA Technical Report 86-C-2, January 1986.

27. R.T. Bhatt and R.E. Phillips, Mechanical Behavior of SiC Fiber Reinforced Reaction Bonded Silicon Nitride Matrix Composite Laminates, to be published in J. COMP. MATERIALS. 
28. R.T. Bhatt, Oxidation Effects on the Mechanical Properties of a SiC-FiberReinforced Reaction-Bonded $\mathrm{Si}_{3} \mathrm{~N}_{4}$ MatrixComposite, J. AM. CERAM. SOC. 75 [2] 406-12 (1992).

29. N.D. Corbin, G.A. Rossetti, and S.D. Hartline, Microstructure/Property Relationships for SiC Filament-Reinforced RBSN, CERAM. ENG. SCI. PROC. 7 [7-8] 958-968 (1986).

30. N.D. Corbin, C.A. Willkens, and S.D. Hartline, RBSN Matrix Composites Reinforced with Polymer Derived Fibers, to be published in NASA Conference on Metal Matrix. Carbon, and Ceramic Matrix Composites Proceedings, Cocoa Beach, FL, January 1987.

31. N.D. Corbin, C.A. Willkens, and S.D. Hartline, The Influence of Interfacial Modifiers on RBSN Matrix Composite Properties, to be published in NASA Conference on Metal Matrix. Carbon, and Ceramic Matrix Composites Proceedings, Cocoa Beach, FL, January 1987.

32. J.S. Haggerty, A. Lightfoot, J.E. Ritter, P.A. Gennari, and S.V. Nair, Oxidation and Fracture Strength of High-Purity Reaction-Bonded Silicon Nitride, J. AM. CERAM. SOC. 72 [9] 1675-79 (1989).

33. A.G. Evans and R.W. Davidge, The Strength and Oxidation of ReactionSintered Silicon Nitride, J. MATER. SCI. 5, 314-25 (1970).

34. R.W. Davidge, A.G. Evans, D. Gilling, and P.R. Wilyman, Oxidation of Reaction Sintered Silicon Nitride and Effects in Strength, pp. 329-43 in Special Ceramics, Vol. 5, Proceedings of the 5th International Symposium on Special Ceramics, Stoke-on-Trent, UK, July 1970. Edited by P.

Popper. British Ceramic Research Association, Manchester, UK, 1972.

35. H. Jakus, J.E. Ritter, and W.P. Rogers, Strength of Hot-Pressed Silicon Nitride after High-Temperature Exposure, J.AM. CERAM. SOC., 67 [7] 47175 (1984).

36. Y. Yasutomi, H. Kita, K. Nakamura, and M. Sobue, Development of Highstrength $\mathrm{Si}_{3} \mathrm{~N}_{4}$ Reacton-bonded SiC Ceramics, J. CERAM. SOC. JPN. INTER. ED. 96, 762-766 (1988).

37. B.W. Sheldon, The Formation of Reaction Bonded Silicon Nitride from Silane Derived Silicon Powders, D.Sc. Thesis, Massachusetts Institute of Technology, February 1989.

38. Y.N. Volgin, and Y.I. Ukhanov, OPT. SPECTROSCOPY 38, 412 (1975).

39. C.P. Gazzara, and D.R. Messier, Determination of Phase Content of $\mathrm{Si}_{3} \mathrm{~N}_{4}$ by $X$-ray Diffraction Analysis, BULL. AM. CERAM. SOC. 56, 777-80 (1977).

40. B.W. Sheldon, J. Szekely, and J.S. Haggerty, Formation of ReactionBonded Silicon Nitride from Silane-Derived Silicon Powders: Macroscopic Kinetics and Related Transport Phenomena, J. AM. CERAM. Soc. 75 [3] 677-85 (1992).

41. M.B. Fegley, The Thermodynamic Properties of Silicon Oxynitride, J. AM. CERAM. SOC. 64, C124-C126 (1981).

42. A.H. Heuer, and V.L.K. Lou, Volatility Diagrams for Silica, Silicon Nitride, and Silicon Carbide and Their Application to High-Temperature Decomposition and Oxidation, J. AM. CERAM. SOC. 73, 2785-3128 (1990). 
43. B.W. Sheldon, and J.S. Haggerty, Nitrogen adsorption onto silane derived silicon powders, J. AM. CERAM. SOC. 74, 1417-24 (1991).

44. R.V. Giridhar, and K. Rose, Conditions for Thermal Nitridation of Si in $\mathrm{N}_{2}-$ $\mathrm{O}_{2}$ Mixtures, J. ELECTROCHEM. SOC. 135, 2803-07 (1988).

45. H. Du, R.E. Tressler, and K.E. Spear, Thermodynamics of the Si-N-O System and Kinetic Modeling of Oxidation of $\mathrm{Si}_{3} \mathrm{~N}_{4}$, J. ELECTROCHEM. SOC. 136, 3210-15 (1989).

46. R.S. Parikh, A. Lightfoot, J.S. Haggerty, and B.W. Sheldon, The Effects of Trace $\mathrm{O}_{2}$ Levels on the Nitriding Kinetics of High Purity Si Powders to Form Reaction Bonded Silicon Nitride, to be submitted to J. AM. CERAM. SOC.

47. A. Lightfoot, R.S. Parikh, J.S. Haggerty, and B.W. Sheldon, Nitridation of Binder and Solvent Exposed High Purity Silicon Powder, submitted to J. AM. CERAM. SOC.

48. QPACTM product literature, Air Products and Chemicals, Inc., Emmaus, PA 18049.

49. R.T. Bhatt, Effects of Fabrication Conditions on the Properties of SiC Fiber Reinforced Reaction-Bonded Silicon Nitride Matrix Composites (SiC/RBSN), NASA Technical Memorandum 88814, January 1986.

50. D. Seyferth, and G.H. Wiseman, High-Yield Synthesis of $\mathrm{Si}_{3} \mathrm{~N}_{4} / \mathrm{SiC}$ Ceramic Materials by Pyrolysis of a Novel Polyorganosilazane, J. AM. CERAM. SOC. 67 [7] C-132-3 (1984).

51. A. Lightfoot, B.W. Sheldon, J.H. Flint, and J.S. Haggerty, Nitriding Kinetics of Si-SiC Powder Mixtures as Simulations of Reaction Bonded $\mathrm{Si}_{3} \mathrm{~N}_{4}-\mathrm{SiC}$ Composites, Ceram. ENG. SCI. Proc. 10 [9-10] 1035-48 (1989).

52. A. Lightfoot, J. Sigalovsky, and J.S. Haggerty, Analyses of RBSN Having High and Low Fracture Toughnesses, to be submitted to J. AM. CERAM. Soc.

53. S.V. Nair, Z.Q. Cai, J.E. Ritter, A. Lightfoot, and J.S. Haggerty, Mechanical Behavior of Silicon Carbide Particulate Reinforced Reaction Bonded Silicon Nitride Matrix Composites, 16th Annual Conference on Composites and Advanced Ceramics, American Ceramic Society, Cocoa Beach, FL, January 1992, published in CERAM. ENG. SCI. PROC. 13 [7-8] 81-89 (1992).

54. Ervin E. Underwood, Quantitative Metallography, in Volume 9, Metallography and Microstructures of Metals Handbook, 9th Edition, 1978.

55. Professor Lisa C. Klein, Rutgers University, private communication

56. W.D. Kingery, H.K. Bowen, and D.R. Uhlmann, Introduction to Ceramics, 2nd edition, John Wiley \& Sons, New York, pp. 528-530. 


\section{Attachment I. List of Publications Resulting in part from DOE Support}

1. Sheldon, Brian W., The Formation of Reaction Bonded Silicon Nitride from Silane Derived Silicon Powders, Sc.D. Thesis, Massachusetts Institute of Technology, 1989.

Reaction bonded silicon nitride (RBSN) was formed from laser synthesized, submicron silicon powders. Complete or nearly complete conversion to $\mathrm{Si}_{3} \mathrm{~N}_{4}$ was obtained at low temperatures and in relatively short times $(e . g .<$ 5 hours at $1100^{\circ} \mathrm{C}$ and $<10$ minutes at $1250^{\circ} \mathrm{C}$ ), compared to the multiday firing schedules at temperatures above $1400^{\circ} \mathrm{C}$ which are used to produce RBSN from conventional oxidized powders. This result is particularly promising for using RBSN as a composite matrix, because the severity of conventional firing schedules typically degrades the reinforcement material (whiskers, fibers, etc.). The rapid nitriding rates were sensitive to submonolayer surface contamination caused by either oxidation or by alcohol exposure during colloidal processing. Research into the mechanisms which control this process was focused on macroscopic transport phenomena, and on developing a detailed description of the nitriding reaction at a particle level.

Samples nitrided in a conventional high purity nitrogen atmosphere $(<10$ ppm total impurities) exhibit gradients where there was more conversion to $\mathrm{Si}_{3} \mathrm{~N}_{4}$ at the sample centers than near the outer surfaces. Experimental results and analysis of the relevant transport processes show that heat transfer and the diffusion of nitrogen and silicon vapor species can create significant gradients, but that the predominant cause of incomplete conversion near the outer surfaces is oxygen contamination from the nitriding gas. Complete conversion was obtained by lowering the oxygen content of the gas to eliminate the composition gradients.

Four distinct stages during nitridation were identified by thermogravimetric analysis (TGA). Between 700 and $1000^{\circ} \mathrm{C}$ the chemisorbed hydrogen monolayer was converted to a chemisorbed nitrogen monolayer. The sigmoidal reaction curve (weight gain versus time) occurs because of a multistep reaction involving both hydrogen adsorption and nitrogen desorption. When the adsorption step was isolated after a vacuum hold to remove the surface hydrogen, the adsorption kinetics obeyed a simple first order rate law, with an activation energy of $140 \mathrm{~kJ} / \mathrm{mole} \mathrm{N}$, and a relatively low sticking probability of $10^{-12}$ to $10^{-10}$.

Above $1000^{\circ} \mathrm{C}$, nitridation was initially very slow; during this induction period crystalline material was not detected by FTIR. A "fast" reaction period was iritiated by the nucleation of crystalline $\mathrm{Si}_{3} \mathrm{~N}_{4}$; this leads to complete conversion at temperatures above $1100^{\circ} \mathrm{C}$, when the surface is 
not contaminated. TEM results show that silicon nitride forms by the nucleation and growth of individual crystallites, rather than by the formation of a coherent product layer. The crystallites which form typically exhibit preferred growth along the [0001] $\mathrm{Si}_{3} \mathrm{~N}_{4}$ axis. Nucleation occurs with a number of different $\mathrm{Si}$ and $\mathrm{Si}_{3} \mathrm{~N}_{4}$ planes, but there is some tendency for nucleation to occur with the $\{11 \overline{2} 0\} \mathrm{Si}_{3} \mathrm{~N}_{4}$ (both $\alpha$ and $\beta$ ) interplanar spacings coincident with $\{110\}$ Si spacings, although one counter example was observed.

The rapid reaction rates were close to the approximate silicon evaporation rates which were measured, suggesting that this process is at least partially rate controlled by silicon evaporation and condensation, although silicon surface diffusion and nitrogen transport through the pore structure may also be relevant. The nitrogen reactivity in this higher temperature range (compared to chemisorption results below $1000^{\circ} \mathrm{C}$ ) apparently increases, with a sticking probability between $10^{-11}$ and $10^{-5}$. At low temperatures or with contaminated surfaces, incomplete conversion to $\mathrm{Si}_{3} \mathrm{~N}_{4}$ occurs because an insufficient number of nuclei form. Slower nitridation after the "fast" reaction apparently involves the growth of non-preferred $\mathrm{Si}_{3} \mathrm{~N}_{4}$ interfaces, and/or the direct nitridation of the solid silicon.

Complete conversion below $1300^{\circ} \mathrm{C}$ was only obtained during the "fast" reaction. For conditions where incomplete conversion was observed, $\mathrm{Si}_{3} \mathrm{~N}_{4}$ formation can be increased by raising the nucleation rate per unit volume. This was achieved by decreasing the particle size. It should also be possible to achieve higher conversion by altering the surface chemistry, although several attempts to do this were unsuccessful.

2. Sheldon, B.W. and J.S. Haggerty, The Nitridation of High Purity, LaserSynthesized Silicon Powder to Form Reaction Bonded Silicon Nitride, Ceram. Eng. SCI. Proc. 9 [7-8] 1061-71 (1988).

The nitriding kinetics of high purity $0.2 \mu \mathrm{m}$ Si powders made from laser heated $\mathrm{SiH}_{4}$ gas were studied with thermogravimetric analysis (TGA), $X-$ ray diffraction, scanning electron microscopy (SEM), and transmission electron microscopy (TEM). Compared to conventional reaction bonded $\mathrm{Si}_{3} \mathrm{~N}_{4}$ (RBSN), nitridation occurred more rapidly and at lower temperatures (e.g. $>97 \%$ conversion in less than ten minutes at $1250^{\circ} \mathrm{C}$ ). Samples exhibited unusual reverse reaction gradients with more nitridation occurring inside of pellets than at the outer surfaces. Exposure to methanol retarded nitridation.

3. Lightfoot, A., B.W. Sheidon, J.H. Flint, and J.S. Haggerty, Nitriding Kinetics of Si-SiC Powder Mixtures as Simulations of Reaction Bonded $\mathrm{Si}_{3} \mathrm{~N}_{4}-\mathrm{SiC}$ Composites, Ceram. ENG. SCI. Proc. 10 [9-10] 1035-48 (1989). 
The nitriding kinetics of $\mathrm{Si}$ and $\mathrm{Si}$ plus $\mathrm{SiC}$ powder mixtures were studied to simulate the fabrication of RBSN-SiC ceramic matrix composites. Very clean, as-synthesized and solvent-exposed powders were studied; C-rich

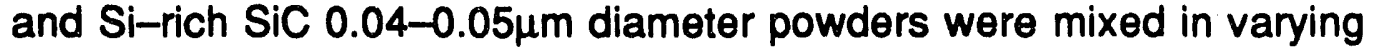
concentrations with $\mathrm{SiH}_{4}$-derived $0.2-0.3 \mu \mathrm{m}$ diameter $\mathrm{Si}$ powder.

Reactions were monitored with TGA and samples were characterized by SEM and $X$-ray diffraction. Although all exposures inhibited the nitriding kinetics from rates observed with the as-synthesized Si powders, complete conversion was still achieved with nitriding schedules that permit fabrication of composites. For example, complete nitridation is achieved with $\mathrm{C}$-rich SiC powders in 140 minutes at $1250^{\circ} \mathrm{C}$, and in the centers of Si-rich SiC powders in 15 minutes. The effects on the incubation periods, fast reaction periods and slow reaction periods that characterize these nitriding processes were studied to explain unusual reverse reaction gradients and other effects of contaminations.

4. Sheldon, B.W., and J.S. Haggerty, The Formation of Reaction Bonded $\mathrm{Si}_{3} \mathrm{~N}_{4}$ at Low Temperatures and in Short Times, CERAM. ENG. SCI. Proc. 10 [7-8] 784-793 (1989).

The kinetics and mechanisms of nitriding high purity, $\mathrm{SiH}_{4}$ derived $\mathrm{Si}$ powders were investigated. TGA, $X$-ray diffraction, FTIR spectroscopy, and TEM techniques were employed. Complete nitridation ( $>99 \%$ ) was achieved at $1250^{\circ} \mathrm{C}$ in less than 10 minutes, and nearly complete nitridation $(97 \%)$ was achieved at $1100^{\circ} \mathrm{C}$ in less than 5 hours. The effects of temperature, particle diameter, and surface chemistry were studied and results show that the process is principally controlled by the number of $\mathrm{Si}_{3} \mathrm{~N}_{4}$ nuclei that form. Reduced temperature, increased Si particle diameter, and surface contamination all reduce the conversion rate through direct effects on nucleation.

5. Haggerty, J.S., High Performance, Porous, Brittle Structural Ceramics, 37th Sagamore Conference, on Structural Ceramics, Plymouth, MA, October 1990, published in Sagamore Army Materials Research Conference Proceedinas, pp. 307-316.

Traditionally with brittle materials, acceptable properties have only been achieved with low porosities. With very few exceptions, the thrust of ceramics processing research has been one of minimizing both porosity and grain size while achieving a desired phase chemistry. Sintering aids, that degrade high temperature performance, are usually an essential feature of this strategy.

Our results with reaction bonded silicon nitride (RBSN) show that porous brittle materials can exhibit properties that equal or exceed those of their fully dense counterparts provided that they are made with the same ievel of 
perfection as is sought in the traditional materials. With over $25 \%$ interconnected porosity, we have achieved strengths up to $870 \mathrm{MPa}$, oxidation resistances up to 20 times lower than fully dense $\mathrm{Si}_{3} \mathrm{~N}_{4}$, and no loss of strength after oxidizing exposures. Strength-to-weight and strainto-failure values for these materials exceed those of fully dense $\mathrm{Si}_{3} \mathrm{~N}_{4}$. These results are understood in terms of fracture mechanics and the uniquely high purities that can be achieved with reaction formed ceramics.

The RBSN results serve as a model for other materials and reveal opportunities for making ceramic parts based on processes that have been avoided because they tend to leave levels of porosity that have been considered unacceptable. These net-shape processes appear capable of producing unique parts with equal or improved properties. Examples from our own work as well as of polymer derived and composite parts will be discussed.

6. Haggerty, J.S., A. Lightfoot, J.E. Ritter, and S.V. Nair, High Strength, Porous, Brittle Materials, MAT. RES. SOC. SYMP. PROC. Vol. 207, pp. 71-76 (1991).

Contrary to existing models, strengths need not be a strong function of porosity for intremediate density, brittle materials. Flaw sizes can remain small $(<50 \mu \mathrm{m})$ if the void space is distributed uniformly in minimum dimension pores. For RBSN, we have demonstrated that fracture toughness can decrease at most linearly with porosity for $0<$ porosity $<40 \%$. Strains to failure and specific strengths of these materials are better than fully dense counterparts.

7. Sheldon, B.W., and J.S. Haggerty, Nitrogen Adsorption onto Silane Derived Silicon Powders, J. AM. CERAM. SOC. 74 [6] 1417-24 (1991).

Nitrogen chemisorption onto silane-derived silicon powder was studied at atmospheric pressure in $\mathrm{N}_{2}$, using thermogravimetric analysis (TGA) to examine the reaction kinetics and using Fourier transform infrared (FTIR) spectroscopy to characterize the surface chemistry. Above $700^{\circ} \mathrm{C}$ the initial $\mathrm{Si}-\mathrm{H}$ surface is transformed to an $\mathrm{Si}-\mathrm{N}$ surface; the latter is characterized by a broad infrared absorption band at 830 to $840 \mathrm{~cm}^{-1}$. Isothermal TGA data exhibit a sigmoidal shape which is apparently caused by simultaneous hydrogen desorption and nitrogen adsorption. By holding samples under vacuum at $900^{\circ} \mathrm{C}$ before nitridation the adsorption reaction was isolated. The kinetics of this reaction are well described by a sample first-order model with an activation energy of $140 \mathrm{~kJ} / \mathrm{mol}$, and with very low sticking probabilities (between $10^{-13}$ and $10^{-10}$ ).

8. Sheldon, B.W., J. Szekely, and J.S. Haggerty, Formation of ReactionBonded Silicon Nitride from Silane-Derived Silicon Powders: Macroscopic 
Kinetics and Related Transport Phenomena, J. AM. CERAM. SOC. 75 [3] 677-85 (1992).

Reaction-bonded silicon nitride (RBSN) with little or no residual silicon was formed from laser-synthesized silicon powders and $\mathrm{N}_{2}$, at low temperatures and in short times $\left(<10\right.$ minutes at $1250^{\circ} \mathrm{C},<1$ hour at $\left.1150^{\circ} \mathrm{C}\right)$. The nitriding kinetics were studied with thermogravimetric analysis (TGA). Samples formed in the TGA furnace between 1150 and $1350^{\circ} \mathrm{C}$ were completely converted to silicon nitride near their centers, with residual silicon near the external surfaces. These unusual composition profiles were not observed in samples that were formed in a higher purity atmosphere. Various experimental observations were compared to analyses of heat and mass transport, and it was determined that these composition gradients were probably caused by oxygen contamination in the nitriding gas. These analyses also indicate that heat transfer, $\mathrm{N}_{2}$ diffusion, and $\mathrm{Si}$ evaporation and transport all affect the nitriding kinetics under certain conditions.

9. Lightfoot, A., R.S. Parikh, J.S. Haggerty, and B.W. Sheldon, Nitridation of Binder and Solvent Exposed High Purity Silicon Powder, submitted to J. AM. Ceram. SOC.

High purity, submicron Si powders having hydrogenated surfaces made from $\mathrm{SiH}_{4}$ gas nitride rapidly at unusually low temperatures. Exposures to polymers required for fabrication of parts alter the powders' surface chemistry directly through reactions with the polymers, or indirectly by making them vulnerable to effects made possible by elevated temperatures required for binder removal. The nitriding kinetics of $\mathrm{SiH}_{4}$ derived $\mathrm{Si}$ powders exposed to polyethylene, polystyrene, $\mathrm{Qpac}^{\mathrm{TM}}$, tetrafluoroethylene/polystyrene and polysilazane binders have been studied. The surface chemistries of the powders after binder burnout in $\mathrm{N}_{2}$ or vacuum were related to nitriding kinetics, and nitrided samples were characterized. Relatively long exposures to temperatures required for binder burnout are the primary cause for retardation or inhibition of the nitriding reactions through the effects of oxygen contamination; however, polymer residues can either accentuate or negate this effect. Simple hydrocarbon polymers, polyethylene and polystyrene, left a carbon residue ( $\mathrm{Si}-\mathrm{C}$ and $\mathrm{Si}-\mathrm{CH}_{3}$ bonding) which offset the inhibiting effect of oxygen to permit > $90 \%$ conversion after slightly extended induction periods. The others left more complex residues which extended the induction period, suppressed the fast reaction rate period, and caused premature termination of the fast reaction period. Exposure to tetrafluoroethylene had the most inhibiting effect and apparently caused Si losses. Each polymer had a different effect on the nucleation of $\mathrm{Si}_{3} \mathrm{~N}_{4}$. SiC fibers were shown to have a negligible effect on the Si nitriding kinetics. 
10. Parikh, R.S., A. Lightfoot, J.S. Haggerty, B.W. Sheldon, The Effects of Trace $\mathrm{O}_{2}$ Levels on the Nitriding Kinetics of High Purity Si Powders to Form Reaction Bonded Silicon Nitride, to be submitted to J. AM. CERAM. SOC.

The effects of trace $\mathrm{O}_{2}$ levels on the nitridation of compacts made from silane- derived Si powders were studied in $\mathrm{N}_{2}$ atmospheres, with oxygen levels of either $5 \mathrm{ppm}$ or $10 \mathrm{ppb}$ (approximately). The nitriding kinetics were studied by thermogravimetric analysis as a function of temperature $\left(1100^{\circ} \mathrm{C}-1200^{\circ} \mathrm{C}\right)$ and heating rate $\left(5^{\circ} \mathrm{C} / \mathrm{min}\right.$ and $\left.100^{\circ} \mathrm{C} / \mathrm{min}\right) . X$-ray diffraction was used to determine the phase composition. Reducing the $\mathrm{O}_{2}$ level in the nitriding gas enhanced conversion to $\mathrm{Si}_{3} \mathrm{~N}_{4}$ at lower temperatures, reduced the composition variations across the samples, and decreased the $\alpha / \beta$ ratios. The results indicate that the rapid formation of $\mathrm{Si}_{3} \mathrm{~N}_{4}$ at relatively low temperatures is only possible under conditions that permit active oxidation of the silicon surfaces.

11. Lightfoot, A., L. Ewart, J. Haggerty, Z.Q. Cai, J. Ritter and S. Nair, Processing and Properties of SiC Whisker and Particulate Reinforced Reaction Bonded $\mathrm{Si}_{3} \mathrm{~N}_{4}$, Ceram. ENG. SCI. Proc. 12 [7-8] 1265-1291 (1991).

Intermediate density, high purity RBSN made from silane-derived $\mathrm{Si}$ powders has a specific st. ength and strain-to-failure higher than fully dense $\mathrm{Si}_{3} \mathrm{~N}_{4}$. Because the fracture toughness is comparatively low, this research sought to increase fracture resistance through composite toughening with particulate and whisker additions. The RBSN composites were made from colloidally pressed octanol dispersions of $0.2-0.3 \mu \mathrm{m}$ diameter, high purity Si powders mixed with either SiC whiskers, $\mathrm{SiC}(\mathrm{w})$, or Acheson $\alpha-\operatorname{SiC}$ particles, $\operatorname{SiC}(p)$, having diameters of $\sim 0.5 \mu \mathrm{m}$ and $\sim 2.0$ $\mu \mathrm{m}$, respectively. The effect of the reinforcements on room temperature strength, fracture toughness and R-curve behavior, and interactions between cracks and reinforcements were studied. None of the materials exhibited R-curve behavior. While the microstructural properties of the matrix were retained and no evidence of $\mathrm{SiC}$ surface degradation was observed, an increase in $\mathrm{K}_{\mathrm{c}}$ was seen only in the 33 vol\% $\mathrm{SiC}(\mathrm{p})$. Details of the fracture process were revealed by SEM of surface cracks and fracture surfaces as well as TEM. Inoperative toughening mechanisms in the whisker composite were attributed to non-uniform whisker morphology. The modest increase in $\mathrm{K}_{\mathrm{C}}$ of the 33 vol\% particulate samples resulted from crack deflection around larger particles.

12. Haggerty, J.S., Synthesis of Reaction Bonded Silicon Nitride from High Purity Si Powders, Materials Research Society 1991 Fall Meeting, Boston, MA, December 1991, published in MAT. RES. SOC. SYMP. PROC. Vol. 249, pp. 495-509. 
Significantly improved reaction kinetics and properties have been realized for reaction bonded $\mathrm{Si}_{3} \mathrm{~N}_{4}$ (RBSN) by using Si powders having unusual physical and chemical characteristics combined with clean, oxygen-free processing conditions. For instance, complete nitridation is achieved in $<1$ $\mathrm{h}$ at $1150^{\circ} \mathrm{C}$ or $<10 \mathrm{~m}$ at $1250^{\circ} \mathrm{C}$ without pre-nucleation, or $<1 \mathrm{~h}$ at $1100^{\circ} \mathrm{C}$ with pre-nucleation. These compare with normal schedules of $\sim 100 \mathrm{~h}$ at $1470-1500^{\circ} \mathrm{C}$. Resulting strengths (up to $870 \mathrm{MPa}$ ) and oxidation resistances of the RBSN essentially equal those of fully dense $\mathrm{Si}_{3} \mathrm{~N}_{4}$ even though RBSN includes $25 \%$ open porosity. The results show that every aspect of the process, ranging from powder synthesis to $<\mathrm{ppm}$ levels of oxygen in the nitriding atmosphere have demonstrable effects on the reaction kinetics and the resulting properties of the parts. With careful processing, reaction formed ceramics can compete advantageously with fully dense counterparts. This paper will review our research on synthesis and properties of RBSN made from $\mathrm{SiH}_{4}$ derived submicron Si powders and make comparisons with conventional RBSN. Powder characteristics will be related to synthesis conditions. The evolution of surface chemistry during nitriding, with and without solvent exposures, will be discussed. The relationship of the $\alpha / \beta$ ratio to nucleation with limited numbers of heteroepitaxial relationships will be shown. These effects will be related to nitriding kinetics. Finally, mechanical and oxidation characteristics will be discussed in terms of the resulting microstructural features and processing defects.

13. Sheldon, B.W., J. Rankin, and J.S. Haggerty, Formation of Reaction Bonded Silicon Nitride from Silane Derived Silicon Powders: Nucleation and Growth Mechanisms, to be submitted to J. AM. CERAM. SOC.

The nucleation and growth of $\mathrm{Si}_{3} \mathrm{~N}_{4}$ on silane derived $\mathrm{Si}$ powders was investigated with transmission electron microscopy and FTIR spectroscopy. Thermogravimetric analysis was used to monitor the process through different stages of the reaction process. FTIR and TEM results provide clear evidence that the nucleation of crystalline $\mathrm{Si}_{3} \mathrm{~N}_{4}$ coincides with the onset of rapid nitridation. TEM and electron diffraction indicate that $\mathrm{Si}_{3} \mathrm{~N}_{4}$ forms heteroepitaxially on the Si powder surfaces. The results reported here demonstrate that the rapid, low temperature nitridation observed with silane derived powders is possible because Si transport occurs through the vapor phase. This is significantly different than the standard model described by Moulson, where the nitridation is limited by solid state diffusion through the $\mathrm{Si}_{3} \mathrm{~N}_{4}$ product layer and incomplete conversion occurs because of slow solid state diffusion. With the powders investigated here, incomplete nitridation was observed at low nucleation rates where there were not enough growth surfaces for rapid $\mathrm{Si}_{3} \mathrm{~N}_{4}$ formation. 
DATE

FILMED

$5 / 12 / 93$ 
